UNIVERSIDADE FEDERAL DO RIO GRANDE DO SUL PROGRAMA DE PÓS-GRADUAÇÃO EM CIÊNCIAS DA SAÚDE:

CARDIOLOGIA E CIÊNCIAS CARDIOVASCULARES

ASSOCIAÇÃO ENTRE A INGESTÃO DE MACRONUTRIENTES E A OBESIDADE ABDOMINAL COM A DOENÇA ARTERIAL CORONÁRIA

MAGDA AMBROS CAMMERER

Porto Alegre 
UNIVERSIDADE FEDERAL DO RIO GRANDE DO SUL PROGRAMA DE PÓS-GRADUAÇÃO EM CIÊNCIAS DA SAÚDE: CARDIOLOGIA E CIÊNCIAS CARDIOVASCULARES

\section{ASSOCIAÇÃO ENTRE A INGESTÃO DE MACRONUTRIENTES E A OBESIDADE} ABDOMINAL COM A DOENÇA ARTERIAL CORONÁRIA

Dissertação apresentada ao Curso de PósGraduação em Ciências da Saúde: Cardiologia e Ciências Cardiovasculares da UFRGS para a obtenção do título de Mestre.

\section{MAGDA AMBROS CAMMERER}

Orientador: Prof. Dr. Waldomiro Carlos Manfroi

Porto Alegre 


\section{AGRADECIMENTOS}

Ao Professor Doutor Waldomiro Carlos Manfroi, por acreditar na potencialidade das pessoas e ser um visionário na medicina.

À coordenação deste curso de pós-graduação, representada pela Professora Doutora Nadine Clausell.

Aos professores deste curso de pós-graduação pela excelência de seu ensino.

Às enfermeiras, técnicos de enfermagem e secretárias da Unidade de Hemodinâmica do Hospital de Clínicas de Porto Alegre, pelo seu apoio durante a etapa de coleta de dados.

À Doutora Lúcia Pellanda, pelo seu incentivo na redação desta dissertação.

À estatística Vânia Naomi Hirakata, pelo seu auxílio nas análises de dados deste trabalho.

À secretária do curso de pós-graduação Sirlei Ferreira Reis, pelas palavras amigas nos momentos difíceis.

Aos meus colegas deste curso de pós-graduação, pelo agradável companheirismo da vida acadêmica.

Às pesquisadoras, Ana Cristina Riehs Camargo, Fernanda da Silva Rodrigues, Márcia Keller Alves e Michele Soares Fraga, cujo esforço e dedicação permitiu a realização deste trabalho. 
Aos meus pais, Ercio e Vivian, pelo dom da vida e pelo estímulo e apoio constantes, em todas as etapas da minha vida.

Ao meu esposo, Fernando, toda a minha gratidão e amor pela vida compartilhada.

Às minhas filhas, Juliana e Isabella, pelo seu sorriso diário e por serem crianças maravilhosas.

Aos meus irmãos, Nelson e Rodrigo, pelo incentivo, mesmo à distância.

Aos meus alunos pelos seus "porquês" constantes.

E finalmente meu agradecimento aos pacientes anônimos, sem os quais este trabalho não seria possível. 


\section{SUMÁRIO}

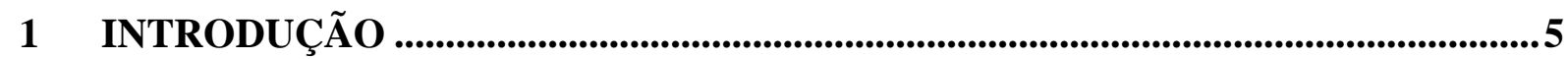

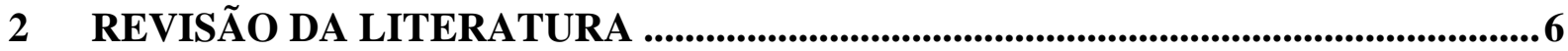

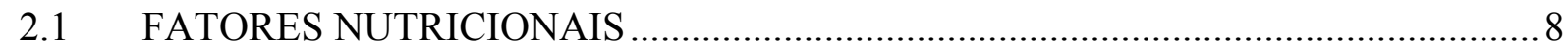

2.2 OBESIDADE E DOENÇA ARTERIAL CORONÁRIA …………………………........ 11

2.3 DISTRIBUIÇÃO DE GORDURA CORPORAL E A DOENÇA ARTERIAL

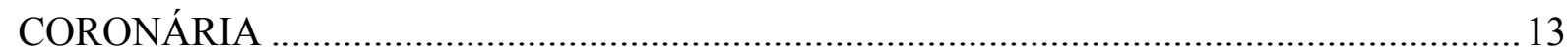

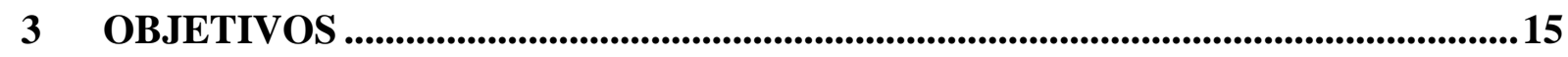

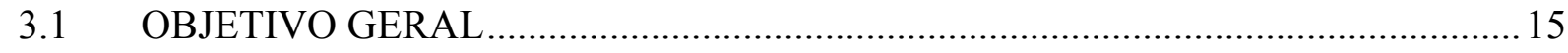

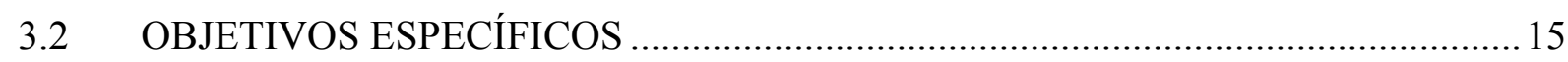

4 REFERÊNCIAS ............................................................................................................. 16

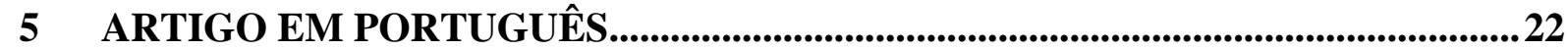

6 ARTIGO EM INGLÊS ....................................................................................................46 


\section{INTRODUÇÃo}

A doença arterial coronária apresenta uma grande incidência na população mundial e continua sendo uma das principais causas de morbidade e mortalidade. Grandes esforços têm sido feitos para a prevenção e o tratamento dessa doença, assim como para a identificação dos fatores de risco.

Dentre os fatores de risco predisponentes para a doença arterial coronária, encontram-se a obesidade e em especial, a obesidade abdominal. Sendo que estas estão diretamente relacionadas com a ingestão de nutrientes e o gasto energético nas atividades diárias.

Neste estudo pretendemos avaliar o consumo diário de nutrientes e sua associação com a presença de obesidade abdominal e outros fatores de risco para a doença arterial coronária, tais como: a idade, a presença de tabagismo, o sexo, o sedentarismo, a presença ou não de diabete melitus e hipertensão arterial, os níveis de colesterol total, o LDL-colesterol, o HDLcolesterol e os triglicerídios. A população escolhida, são pacientes portadores de cardiopatia isquêmica, submetidos a cateterismo cardíaco no Hospital de Clínicas de Porto Alegre. 


\section{REVISÃO DA LITERATURA}

Segundo estudos realizados na Europa, nos Estados Unidos e no Brasil, a incidência da doença arterial coronária (DAC) continua sendo a principal causa de morbidade e mortalidade em adultos. ${ }^{(1-3)}$

De acordo com dados do American Heart Association (AHA) ${ }^{(4)}$, foram realizados avanços quanto ao entendimento da fisiopatologia da doença em relação às medidas de prevenção primária e secundária. Houve, também, um grande desenvolvimento na identificação de fatores causais e na prevenção dos mesmos para evitar os desfechos clínicos e/ou novos eventos, assim como a implementação de novas alternativas terapêuticas, tanto de ordem medicamentosa como procedimentos invasivos.

A intervenção primária na prevenção da morbidade e mortalidade para as doenças cardiovasculares é a redução dos fatores de risco para estas doenças. Estudos epidemiológicos vêm demonstrando que fatores de risco, tais como tabagismo, hipertensão e dislipidemia, agem associadamente. $\mathrm{O}$ conceito de identificação e modificação de fator de risco é baseado na premissa de que a exposição a um grande número de fatores ambientais aumentam o risco de desenvolver a doença, sendo que, a modificação dessas condições reduzem o risco. ${ }^{(5)}$

A identificação dos fatores de risco para a DAC tornou-se muito importante na predição dessa doença, tanto que, nas três últimas décadas, o perfil dos riscos cardiovasculares foi avaliado através de diversos estudos observacionais longitudinais. ${ }^{(6)}$

Esses estudos formaram a base para o desenvolvimento de programas de prevenção das doenças coronárias. O Heart Disease Epidemiology Study of National Heart Institute in Framingham, Massachussetts, foi o primeiro bem-sucedido e detalhado estudo epidemiológico sobre as doenças do coração. ${ }^{(7)}$ 
Nos primeiros editoriais publicados sobre os estudos de Framingham, os autores relatavam que a aterosclerose e as doenças relacionadas eram consideradas resultados inerentes ao processo de envelhecimento. Àqueles que procuravam descobrir as possíveis causas da doença aterosclerótica eram cotados membros do "Culto da Esperança", esforçando-se em encontrar soluções para um problema insolúvel. ${ }^{(7)}$ No entanto, apesar do pessimismo e intensas investigações envolvendo pesquisas clínicas, laboratoriais e epidemiológicas, houveram avanços no entendimento da doença e seus fatores determinantes.

Desde os estudos de Framingham ${ }^{(1)}$, os outros trabalhos vêm demonstrando, de forma sistemática, que os fatores de risco têm um efeito multiplicativo, isto é, indivíduos com dois ou três fatores, de pouca magnitude, podem ter um risco muito maior do que indivíduos com um fator isolado de maior importância. ${ }^{(4)}$

A etiologia da doença arterial coronária é complexa e multifatorial, segundo o AHA e o American College of Cardiology (ACC), os fatores de risco independentes para DAC são tabagismo, hipertensão arterial sistêmica (HAS), colesterol total elevado, lipoproteína de baixa densidade (LDL-C) elevada, lipoproteína de alta densidade (HDL-C) reduzida, diabete mellitus (DM) e idade avançada. ${ }^{(4)}$ Mais tarde, outros fatores foram associados com o aumento do risco para DAC denominando-se fatores de risco condicionantes e predisponentes. ${ }^{(4)}$

A contribuição causal, determinante e quantitativa dos fatores de risco condicionantes ainda não foi bem documentada, porém, sabe-se que estão relacionados com o aumento do risco para DAC. São fatores de risco condicionantes: triglicerídeos elevados, partículas LDL pequenas e densas, homocisteína sérica elevada, lipoproteína(a) sérica elevada, fatores protrombóticos (como fibrinogênio) e marcadores inflamatórios (como proteína C-reativa). ${ }^{(4)}$

Os fatores de risco predisponentes são aqueles que potencializam os fatores de risco independentes. São eles: obesidade ${ }^{(1,4,5,8)}$, obesidade abdominal ${ }^{(9)}$, sedentarismo ${ }^{(10)}$, história familiar de DAC prematura em parentes de primeiro grau, características étnicas e fatores psicossociais. $^{(4)}$

Muitos desses fatores podem ser influenciados por um ou mais componentes alimentares, entretanto, uma vez que a alimentação diária é complexa e contém diversos nutrientes e alimentos, ainda não foi possível elucidar ou quantificar precisamente o impacto específico da alimentação no risco de desenvolver a doença arterial coronária. 


\section{$2.1 \quad$ FATORES NUTRICIONAIS}

No século passado, estudos epidemiológicos e observacionais estabeleceram que a alimentação é um fator importante que pode ajudar a explicar as variações entre os índices de doenças cardiovasculares em diferentes populações. Os primeiros estudos, como o Seven Countries Study ${ }^{(1)}$, identificaram uma relação entre o consumo elevado de calorias provenientes das gorduras saturadas e do colesterol, bem como maior risco de desenvolver DAC. ${ }^{(12)}$

Estudos observacionais demonstraram que populações com pouca incidência de cardiopatia isquêmica consumiam dietas pobres em gordura total, gordura saturada e colesterol; dietas ricas em fibras vegetais e adequadas em relação às proteínas e aos carboidratos. ${ }^{(13,14)}$

O Lyon Diet Heart Study ${ }^{(13)}$, um ensaio sobre a prevenção secundária, em grande escala, avaliou se a dieta mediterrânea "Fase I" conseguiria reduzir a taxa de recorrência após um primeiro infarto. Esse estudo mostrou que os pacientes portadores de cardiopatia isquêmica, que seguiram a dieta mediterrânea "Fase I", que era rica em ácidos alfa-linolênicos, apresentaram uma taxa de mortalidade e morbidade por doença cardiovascular (DCV), 70\% menor do que os pacientes do grupo controle, que receberam apenas orientação nutricional, realizada por médico ou nutricionista. Entre outros resultados, verificou-se que os grupos avaliados não apresentaram diferença entre os níveis de lipídeos séricos. Sugerindo, que a alimentação adequada, completa e variada, oferece os nutrientes necessários para a manutenção da saúde, contrapondo, com a ingestão dos nutrientes isoladamente.

Outro estudo recente, o GISSI-Prevenzione ${ }^{(15)}$ mostrou os benefícios de um único componente alimentar, os ácidos graxos poliinsaturados essenciais (PUFA), ômega-3 e EPA (ácido eicosapentaenóico), presentes no óleo de peixe analisado em uma forma purificada. $\mathrm{O}$ estudo acompanhou pacientes que sobreviveram a um infarto do miocárdio e foram indicados a receber n-3 PUFA e vitamina E. Após o término do estudo, verificou-se que o tratamento com n-3 PUFA, sozinho ou em conjunto com vitamina $E$ efetuou uma redução significativa no índice de óbito por infarto do miocárdio não-fatal e derrame. Os resultados desse estudo, também, não demonstraram alterações detectáveis nos lipídeos do sangue, ou qualquer outro fator de risco estabelecido. 
Em todo o mundo, nunca houve população que ingerisse uma dieta com baixas quantidades de gorduras e que apresentasse altos níveis de colesterol, ou doenças do coração. ${ }^{(11,14)}$ Estes dados foram verificados por estudos clínicos, porém, os resultados são conflitantes quando adotados individualmente.

A partir desses estudos iniciais, foram identificados outros componentes alimentares que têm uma ligação positiva ou negativa com o risco de DCV, entre eles, a composição das gorduras, proteínas, carboidratos, vitaminas, minerais e outras substâncias encontradas nos alimentos. ${ }^{(16)}$

A ingestão diária de gorduras está associada com a obesidade e, por sua vez, relacionada com fatores de risco importantes para a doença arterial coronária. No entanto, esta relação, está associada com o efeito dos ácidos graxos e colesterol da dieta, sobre o colesterol sérico, principalmente o LDL-colesterol.

Devido aos sistemas de retroalimentação que responde ao consumo dietético de colesterol regulando a síntese hepática, o colesterol da dieta, tende a ter um efeito menor sobre o colesterol sérico, se comparado à ingestão de ácidos graxos saturados. No entanto, alguns indivíduos parecem ter um sistema de retroalimentação menos sensível, portanto, que respondem à ingestão de colesterol mais elevada, com níveis séricos rapidamente aumentados. $^{(14)}$

Os ácidos graxos saturados tendem a elevar o LDL-colesterol, um dos mecanismos propostos para essa ação é a redução do número de receptores hepáticos de LDL (receptores B-E), o que pode inibir a remoção plasmática desta partícula e também aumentar os níveis de triglicerídeos no plasma, o que parece decorrer de estímulo na secreção hepática de triglicerídeos sob a forma de VLDL (very low density lipoprotein). ${ }^{(17)}$

Além disso, a gordura saturada, em função da sua estrutura retilínea, permite maior entrada de colesterol nas partículas de LDL. Portanto, a ingestão de gordura saturada é a principal causa alimentar de elevação do colesterol do plasma. ${ }^{(18)}$

A substituição dos ácidos graxos saturados por ácidos graxos poliinsaturados reduz o colesterol total e o LDL-colesterol plasmáticos, por vários mecanismos, sendo os principais: menor produção e maior remoção de LDL-C e alteração da estrutura das LDL de forma a diminuir o conteúdo de colesterol da partícula. Os ácidos graxos poliinsaturados possuem o 
inconveniente de baixar os níveis plasmáticos de HDL-colesterol e de induzir maior oxidação lipídica. Os ácidos graxos monoinsaturados reduzem igualmente o colesterol, sem, no entanto, diminuir o HDL-C e provocar oxidação lipídica. Os ácidos graxos ômega-3 diminuem a trigliceridemia plasmática por reduzirem a secreção hepática de VLDL. ${ }^{(19)}$

Os ácidos graxos trans são sintetizados durante o processo de hidrogenação dos óleos vegetais na produção das margarinas. As moléculas de hidrogênio na posição cis (ácido oléico) produzem configuração com ângulo na cadeia de carbono, enquanto que, na forma trans, a cadeia é retilínea (semelhante à dos ácido graxos saturados). Pela semelhança estrutural com a gordura saturada, a gordura trans também provoca elevação da colesterolemia, com o aumento do LDL-colesterol e redução do HDL-colesterol. ${ }^{(19)}$

Estudos relacionando a qualidade da alimentação consumida ${ }^{(20-23)}$ com a progressão da doença arterial coronária sugerem que o aumento das lesões arteriais está associado com a ingestão de gorduras saturadas e ácidos graxos trans, mesmo sem elevação dos níveis de colesterol plasmático.

De outro modo, pesquisas associando a redução do consumo de gorduras saturadas com a menor incidência de fatores de risco para DAC foram realizadas em diversos países ${ }^{(24-30)}$ e indicam que a redução da ingestão de gorduras saturadas e a substituição para gorduras monoinsaturadas e poliinsaturadas é uma conduta a ser adotada.

Um consumo elevado de carboidratos pode corresponder a um aumento temporário de triglicerídeos séricos e diminuição do HDL-colesterol em indivíduos sensíveis a uma alimentação rica em carboidratos. ${ }^{(31)}$ Essa resposta pode estar relacionada à obesidade, diabete mellitus e intolerância à glicose. ${ }^{(32)}$ Os carboidratos complexos são menos aterogênicos do que os açúcares simples, provavelmente devido às diferenças na resposta à insulina. ${ }^{(33)}$

Alimentos fontes de carboidratos complexos, além de apresentarem na sua composição vitaminas e minerais, também, são fontes de fibras dietéticas. Embora essas fibras possam reduzir o colesterol em indivíduos com altos níveis de lipídeos séricos ${ }^{(34)}$, a evidência de que as fibras possam diminuir o risco de doenças cardiovasculares ainda é inconclusiva. ${ }^{(35-37)}$

As proteínas de origem animal, geralmente contribuem com níveis mais altos de colesterol no sangue e mais aterosclerose do que dietas com proteínas de origem vegetal ${ }^{(27,28)}$, 
provavelmente, porque as proteínas de origem animal são encontradas em alimentos ricos em gorduras saturadas.

No Brasil, estudos de base populacional ${ }^{(38)}$ analisaram a associação entre a dieta habitual e os fatores de risco para a doença arterial coronária em adultos e verificaram que a ingestão diária de proteínas e lipídeos encontra-se acima dos padrões recomendados, enquanto que a ingestão de carboidratos está abaixo das recomendações diárias.

A ingestão excessiva e desequilibrada de macronutrientes e nutrientes calóricos contribui para o sobrepeso, a obesidade e outras doenças associadas.

\subsection{OBESIDADE E DOENÇA ARTERIAL CORONÁRIA}

A obesidade é um distúrbio metabólico cada vez mais prevalente, e está relacionada com um grande número de morbidades, incluindo diversas formas de doenças do coração. ${ }^{8,9)}$ Embora a hereditariedade explique $30 \%$ a $70 \%$ dos casos de obesidade, as contribuições ambientais para o aumento da prevalência da obesidade, tais como a ingestão elevada de nutrientes e a diminuição da atividade física, devem ser analisadas, considerando que as características genéticas mantêm-se estáveis. $^{(8)}$

A relação entre a obesidade e as doenças cardiovasculares já foi bem descrita em diversos estudos prospectivos. ${ }^{(8,9,39,40)}$ Desde os estudos de Framingham, as evidências demonstram que a obesidade está associada também com os outros fatores de risco, como a hipertensão arterial sistêmica, colesterol total, HDL-colesterol, diabete mellitus, fatores protrombóticos e marcadores inflamatórios. ${ }^{(9,41)}$

Kannel e colaboradores demonstraram, em $1967^{(39)}$, que os eventos cardiovasculares eram mais prevalentes nos indivíduos obesos do que aqueles com o peso normal. Também, correlacionaram a maior prevalência de obesidade e DAC em homens, considerando que as mulheres apresentavam poucos desfechos cardíacos. Nesse trabalho, constataram que os eventos cardíacos estavam relacionados com o aumento de peso, e não apenas com a obesidade, sugerindo que o ganho de peso durante a vida do indivíduo era o fator desencadeante para o aumento da pressão arterial e do colesterol sérico. Desde aquela época, 
já sugeriam um programa de redução da ingestão calórica, perda de peso e aumento da atividade física para reduzir a prevalência dos fatores de risco para DAC.

Dietas ricas em gorduras e calorias e um reduzido gasto energético são prováveis explicações para a epidemia de obesidade. Nos EUA, apesar das políticas públicas de incentivo à redução do consumo dos alimentos ricos em gorduras (ao redor de 40\% em 1965 para $34 \%$ em 1991) ${ }^{(42)}$, não houve uma diminuição na incidência de obesidade. Isto pode ser atribuído a duas situações: a manutenção da ingestão das gorduras totais, e a redução da atividade física diária.

No Brasil, estudos que avaliaram a adequação da dieta familiar nas áreas metropolitanas, no período de 1988 e $1996^{(43,44)}$, verificaram que o consumo médio de lipídeos é cerca de $27 \%$ das calorias totais, porém houve um aumento do consumo de ácidos graxos saturados e uma redução do consumo de carboidratos complexos.

Estudos longitudinais, no entanto, indicam que a obesidade está não apenas relacionada, mas também é um preditor independente para a doença arterial coronária. ${ }^{(40,45)}$ Esta relação existe para ambos os sexos, com pequenos aumentos de IMC. Em um estudo prospectivo de 14 anos, avaliando mulheres de meia idade com Índice de Massa Corpórea (IMC) $>23$ e $<25$ $\mathrm{Kg} / \mathrm{m}^{2}$, houve um aumento de $50 \%$ do risco de doença arterial coronária fatal e não fatal. ${ }^{(46)}$ Rimm e colaboradores ${ }^{(45)}$ avaliaram uma coorte de homens de meia idade e idosos (entre 40 e 75 anos) e verificaram o aumento de $72 \%$ do risco para eventos cardiovasculares em homens entre 40 e 65 anos, com IMC entre 25 e $29 \mathrm{Kg} / \mathrm{m}^{2}$.

Recentemente, o PDAY Study (The Pathobiological Determinants of Atherosclerosis in Youth $)^{(40)}$, demonstrou a correlação entre a obesidade e a severidade de lesões ateroscleróticas em um estudo multicêntrico realizado em autópsias de aproximadamente 3000 cadáveres de adultos jovens (15 - 34 anos) que morreram de causas externas.

A definição de obesidade sempre foi controversa e passou por diversos estudos, iniciando com as estatísticas de mortalidade das companhias de seguro norte-americanas, como a "Metropolitan Life Insurance Company", até estudos mais recentes ${ }^{(47)}$, que validaram o Índice de Massa Corpórea (IMC) como um índice adequado para avaliar a obesidade. O IMC é definido pelo peso em quilogramas, dividido pela altura elevada ao quadrado, em centímetros $\left(\mathrm{Kg} / \mathrm{m}^{2}\right){ }^{(48)}$ 
Porém, o IMC não é o melhor preditor para avaliar a distribuição de gordura corporal. ${ }^{(49)}$ Evidências científicas indicam que o aumento da circunferência do abdomen ou a relação cintura/quadril são melhores preditores de comorbidades e mortalidade decorrentes da obesidade. $^{(50-53)}$

\subsection{DISTRIBUIÇÃO DE GORDURA CORPORAL E A DOENÇA ARTERIAL CORONÁRIA}

Diversos estudos que analisaram a relação entre a distribuição da gordura corpórea "versus" o risco para DAC indicaram que a obesidade andróide (gordura na região abdominal) apresenta maior associação com a morbidade e a mortalidade do que a obesidade ginecóide (gordura na região glútea). ${ }^{(50,52,53)} \mathrm{A}$ idade, os hormônios sexuais, o sedentarismo, os fatores genéticos e dietéticos também podem induzir à obesidade abdominal. ${ }^{(45,50,54)}$

$\mathrm{O}$ acúmulo de gordura na região abdominal foi inicialmente descrito por Vague ${ }^{(56)}$ e associado a alterações metabólicas e a doenças cardiovasculares, posteriormente, outros estudos confirmaram esta hipótese ${ }^{(57-59)}$, incluindo distúrbios no metabolismo das lipoproteínas. ${ }^{(60,61)}$

A deposição de gordura abdominal tem alta atividade da lipogênese e lipólise e este acúmulo pode induzir a níveis elevados de ácidos graxos livres na circulação porta. O excesso destes ácidos graxos pode causar uma acentuada síntese de lipídios e ativação da gliconeogênese, assim como desencadear uma resistência à insulina, resultando numa hiperlipidemia, intolerância à glicose, hipertensão e finalmente aterosclerose. ${ }^{(59,61,62,63)}$

Os padrões de deposição de gordura são controlados geneticamente e diferem entre homens e mulheres. O tipo ginóide é mais comum entre mulheres e o tipo andróide é mais comum em homens, porém, padrões dos dois tipos podem ser encontrados em mulheres. ${ }^{(56,64)}$

O melhor método disponível atualmente para avaliar a distribuição de gordura corporal é a tomografia computadorizada, no entanto, essa tecnologia é dispendiosa e requer irradiação sob os indivíduos, limitando o seu uso em estudos clínicos e epidemiológicos. ${ }^{(65)}$ 
No entanto, desde que obesidade abdominal mostrou estar associada com a relação cintura/quadril (RCQ) e a circunferência do abdomen esses índices têm sido largamente utilizados para investigar esta associação.

Estudos de validação da circunferência abdominal ${ }^{(66)}$ recomendam medir a circunferência da cintura no ponto médio, entre a margem da costela inferior e a crista ilíaca, e a circunferência do quadril no ponto mais largo ao redor dos trocânteres maiores. Os pontos de corte utilizados para avaliar circunferência abdominal para homens é $\geq 102 \mathrm{~cm}$ e para mulheres, $\geq 88 \mathrm{~cm}$.

A relação cintura/quadril, obtida através da razão entre a circunferência da cintura e a circunferência do quadril, é simples de ser aplicado e de baixo custo, indicado para avaliar indivíduos em estudos epidemiológicos. ${ }^{(51)}$ Os pontos de corte utilizados para avaliar a RCQ nos homens é $\leq 0,95$ e para as mulheres é $\leq 0,80{ }^{(67)}$

Na década de 90 , diversos estudos ${ }^{(68,69)}$ avaliaram a associação entre a distribuição de gordura corporal, através da circunferência abdominal e da relação cintura/quadril em homens e mulheres, com os achados da angiografia, considerando ser esta a técnica mais sensível para avaliar a doença arterial coronária. Esses trabalhos, demonstraram que o acúmulo de gordura na região abdominal está relacionada com o aumento do risco para desenvolver doenças do coração. Por outro lado, estudos semelhantes verificaram que as mulheres idosas apresentam maior risco para desenvolver a síndrome metabólica do que as mulheres mais jovens ou homens. ${ }^{(70,71)}$

Assim, a partir dessa perspectiva de estudo, na presente pesquisa, pretende-se avaliar a associação entre os hábitos alimentares, a obesidade abdominal e a presença de doença arterial coronária, confirmada pela cinecoronariografia, em um grupo de pacientes do Hospital de Clínicas de Porto Alegre portadores de cardiopatia isquêmica. 


\section{OBJETIVOS}

\subsection{OBJETIVO GERAL}

Avaliar a relação entre o consumo alimentar diário, a obesidade abdominal e achados angiográficos de obstrução arterial em uma população de pacientes portadores de cardiopatia isquêmica, submetidos a cateterismo cardíaco.

\subsection{OBJETIVOS ESPECÍFICOS}

- conhecer os hábitos alimentares e a ingestão de macronutrientes de uma população de pacientes portadores de cardiopatia isquêmica, submetidos a cateterismo cardíaco;

- relacionar os padrões da dieta com a relação cintura/quadril, o Índice de Massa Corpórea e os achados angiográficos;

- avaliar qual dos métodos de predição de obesidade abdominal apresentam maior associação com os resultados anormais do cateterismo cardíaco;

- avaliar a prevalência de fatores de risco para a doença arterial coronária nos indivíduos que compõem a amostra. 


\section{REFERÊNCIAS}

1 Wilson, PWF; D’Agostino, RB; Levy, D; et al. Prediction of coronary heart disease using risk factors categories. Circulation 1998; 97:1837-1847.

2 Grundy, Scott M; Balady, Gary J; Criqui, Michael H e cols. Primary prevention of coronary heart disease: guidance from Framingham. A statement for healthcare professionals from the AHA task force on risk reduction. Circulation 1998;97;1876-87.

3 http://www.datasus.gov.br (31/10/2002).

4 Grundy, SM; Pasternak, R; Greenland, P; et al. Assessment of cardiovascular risk by use of multiple-risk-factor assessment equations. A statement for healthcare professionals from the American Heart Association and the American College of Cardiology. Circulation 1999;100:1481-92.

5 Braunwald, Eugene. Heart disease; textbook of cardiovascular medicine. 5ed. Philadelphia, W B Sauders, 2001.

6 Greenland, P; Grundy, S; Pasternak, MD; Lenfant, C. Problems on the pathway from risk assessment to risk reduction. Circulation 1998;97:1761-1762

7 (Editorial) The Framingham Study. An epidemiological approach to coronary heart disease. Circulation 1966 Oct; 34(4):553-5.

8 Eckel, Robert H; Barouch, Winifred W; Ershow, Abby G. Report of National Heart, Lung, and Blood Institute-National Institute of Diabetes and Digestive and Kidney Diseases Working Group on the Pathophysiology of Obesity-Associated Cardiovascular Disease. Circulation 2002;105:2923-28.

9 Grundy, Scott M. Obesity, metabolic syndrome, and coronary atherosclerosis. Circulation 2002;105:2696-98.

10 Eckel, RH.Obesity and heart disease; a statement for healthcare professionals from the nutrition committee, American Heart Association. Circulation 1997;96:3248-3250. 
11 Keys A, editor. Coronary heart disease in seven countries. Circulation 1970;41(4 suppl 1):I1-I211.

12 Mustad, Vikkie; Kris-Etherton, Penny. Além da redução do colesterol: decifrando os benefícios da intervenção alimentar para a doença cardiovascular. Cur Atheros Rep Brasil 2001;1:2-7.

13 De Lorgeril, M; Salen, P; Martin, J-L, et al. Mediterranean diet, traditional risk factors, and the rate of cardiovascular complications after myocardial infarction: final report of the Lyon Diet Heart Study. Circulation 1999;99:779-85.

14 Mahan, Kathleen \& Escott-Stump, Sylvia. Alimentos, nutrição e dietoterapia. 9 ed. São Paulo, Roca, 1998.

15 Gissi-Prevenzione Investigators: dietary supplementation with n-3 polyunsaturared fatty acids and vitamin E after myocardial infarction: results of the GISSI-Prevenzione trial. Lancet 1999;354:447-55.

16 Hornstra, G; Barth, CA; Galli, C et al. Functional food science and cardiovascular system. Br J Nutr 1998;80(S1):S113-S46.

17 Cuppari, Lilian. Nutrição; nutrição clínica no adulto. Barueri, Manole, p.91, 2002.

18 Krauss, Ronald; Eckel, Robert; Howard, Barbara; Appel, Lawrence; Daniels, Stephen et al. AHA dietary guidelines. Revision 2000: a statement for healthcare professionals from the nutrition committe of the American Heart Association. Circulation 2000;102:2284-99.

19 III Diretrizes brasileiras sobre dislipidemias e diretriz de prevenção da aterosclerose do departamento de aterosclerose da Sociedade Brasileira de Cardiologia. Arq Bras Cardiol 2001:77(supl III).

20 Blankenhorn, DH; Johnson, RL; Mack, WJ; el Zein, HÁ; Vailas, LI. The influence of diet on the appearance of new lesions in human coronary arteries. JAMA 1990;263(12):12512 .

21 Watts, GF; Jackson, P; Burke, V; Lewis, B. Dietary fatty acids and progression of coronary artery disease in men. Am J Clin Nutr 1996;64(2):202-9.

22 Watts, GF; Lewis, B; Jackson, P; Burke, V; Lewis, ES. Et al. Relationships between nutrient intake and progression/regression of coronary atherosclerosis as assessed by serial quantitative angiography. Can J Cardiol 1995;11 suppl G:110G-14G.

23 Watts, GF; Jackson, P; Mandalia, S; Brunt, JN; Lewis, ES et al. Nutrient intake and progression of coronary disease. Am J Clin Nutr 1994;15(5):328-32.

24 Kris-Etherton, PM; Binkoski, AE; Zhao, G; Coval, SM; Clemmer, KF et al. Dietary fat: assessing the evidence in support of moderate-fat diet; the benchmark based on lipoprotein metabolism. Proc Nutr Soc 2002;61(2):287-98.

25 Hooper, L; Summerbell, CD; Higgins, JP; Thompson, RL; Clements, G et al. Reduced or modified dietary fat for preventing cardiovascular disease. Cochrane Database Syst Rev 2000;(2):CD002173. 
26 Caggiula, AW; Mustad, VA. Effects of dietary fat and fatty acids on coronary artery disease risk and total and lipoprotein cholesterol concentrations: epidemiologic studies. Am J Clin Nutr 1997;65(5 Suppl):1597S-1610S.

$27 \mathrm{Hu}, \mathrm{FB}$; Stampfer, MJ; Manson, JE; Rimm, E; Colditz, GA et al. Dietary fat intake and the risk of heart disease in women. N Engl J Med 1997;337(21):1491-9.

$28 \mathrm{Hu}, \mathrm{FB}$; Stampfer, MJ; Manson, JE; Ascherio, A; Colditz, GA et al. Dietary saturated fats and their food sources in relation to the risk of coronary heart disease in women. Am $\mathrm{J}$ Clin Nutr 1999;70(6):1001-8.

29 Posner, BM; Franz, MM; Quatromoni, PA; Gagnon, DR; Sytkowski, PA. Et al. Secular trends in diet and risk factors for cardiovascular disease: the Framingham Study. J Am Diet Assoc 1995;95(2):171-9.

30 Ascherio, A; Rimm, EB; Giovanucci, EL; Spiegelman, D; Stampfer, M. et al. Dietary fat and risk of coronary heart disease in men: cohort follow up study in the United States. BMJ 1996;313(7049):84-90.

31 Blum, CB; Levy, RI. Current therapy for hypercholesterolemia. JAMA 1989; 261:3582.

32 Committe on diet and health, food and nutrition board. National research council: diet and health. Implications for reducing chronic disease risk. Washington, DC, National Academy Press, 1989.

33 Howard, Barbara V; Wylie-Rosett, Judith. Sugar and cardiovascular disease. A statement for healthcare professionals from the committe on nutrition of the council on nutrition, physical activity, and metabolism of the American Heart Association. Circulation 2002;106:523-27.

34 Wurch, P; Pi-Sunyer, FX. The role of viscous soluble fiber in the metabolic control of diabetes. Diabetes Care 1997;20:1774-80.

35 Truswell, AS. Cereal grains and coronary heart disease. Eur J Clin Nutr 2002;56(1):1-14.

36 McKeown, NM; Meigs, JB; Liu, S; Wilson, PW; Jacques, PF. Whole-grain intake is favorably associated wthi metabolic risk factors for type 2 diabetes and cardiovascular disease in the Framingham Offspring Study. Am J Clin Nutr 2002;76(2):390-8.

37 Jacobs, DR Jr; Meyer, KA; Kushi, LH, et al. Whole grain intake may reduce risk of coronary heart disease death in post menopausal women: the Iowa Women's Health Study. Am J Clin Nutr 1998;68:248-57.

38 Cervato, AM; Mazzilli, RN; Martins, IS; Marucci, MFN. Dieta habitual e fatores de risco para doenças cardiovasculares. Rev Saúde Pública 1997;31(3):227-35.

39 Kannel, William; LeBauer, Joseph; Dawber, Thomas R; McNamara, Patricia. Relation of body weight to development of coronary heart disease. The Framingham Study. Circulation 1967 Apr;35(4):734-44. 
40 McGill, Henry Jr; McMahan. Alex; Herderick, Edward E; Zieske, Arthur; Malcom, Gray T. et al. Obestity accelerates the progression of coronary atherosclerosis in young men. Circulation 2002;105:2712-18.

41 Rosito, Guido Bernardo Aranha. Análise da associação entre níveis de fibrinogênio, fatores hemostáticos e redução do potencial fibrinolítico com o aumento da massa corporal e o acúmulo abdominal de gordura no Estudo dos Descendentes de Framingham, Tese (Doutorado) - Universidade Federal do Rio Grande do Sul. Faculdade de Medicina. Curso de Pós-graduação em Cardiologia, 1999, XIII, 110 p.

42 Popkin, BM; Siega-Riz, AM; Haines, OS. A comparison of dietary trends among racial and socioeconomic groupsin the United States. N Engl J Med 1996;335:716-20.

43 Mondini, Lenise; Monteiro, Carlos A. Mudanças no padrão de alimentação da população urbana brasileira (1962-1988). Rev Saúde Pública 1994;28(6):433-9.

44 Monteiro, Carlos Augusto; Mondini, Lenise; Costa, Renata, BL. Mudanças na composição e adequação nutricional da dieta familiar nas áres metropolitanas do Brasil (1988-1996). Rev Saúde Pública 2000;34(3):251-8.

45 Rimm, EB; Stampfer, MJ; Giovannucci, E; et al. Body size and fat distribution as predictor of coronary heart disease among middle-aged and older US men. Am J Epidemiol, 1995; 141(12):1117-27.

46 Manson, JE; Willett, WC; Stampfer, MJ; Colditz, GA; Hunter, DJ. Et al. Body weight and mortality among women. N Engl J Med 1995;333:677-85.

47 Must, A; Dallal, GE; Dietz, WH. Reference data for obesity: $85^{\text {th }}$ and $95^{\text {th }}$ percentiles of body mass index (wt/ht $\left.{ }^{2}\right)$ and triceps skinfold thickness. Am J Clin Nutr 1991;53:839-46.

48 Garrow JS; Webster, J. Quetelet's index $\left(\mathrm{W} / \mathrm{H}^{2}\right)$ as a measure of fatness. Intern J Obes 1985;9:147-53.

49 Kannel, William B; Cupples, Adrienne; Ramaswami, Ratna; Stokes, Joseph III; Kreger, Bernard E. et al. Regional obesity and risk of cardiovascular diseasae; the Framingham Study. J Clin Epidemiol 1991;44(2):183-90.

50 Larsson, B; Svärdsudd, K; Welin, L; Wilhelmsen, L; Björntorp, P; Tibblin, G. Abdominal adipose tissue distribution, obesity, and risk of cardiovascular disease and death: 13 year follow up of participants in the study of men born in 1913. BMJ 1984;288:1401-04.

51 Björntorp, P. Regional patterns of fat distribution. Ann Intern Med 1985;103(6 pt 2):9945.

52 Rexrode, KM; Buring, JE; Manson, JE. Abdominal and total adiposity and risk of coronary heart disease in men. Intern J Obes 2001;25:1047-56.

53 Gustat, J; Elkasabany, A; Srinivasan, S; Berenson, G. Relation of abdominal height to cardiovascular risk factors in young adults. The Bogalusa Heart Study. Am J Epidemiol 2000;151:885-91. 
54 Seidell, Jacob C; Pérusse, Louis; Després, Jean-Pierre; Bouchard, Claude. Waist and hip circumferences have independent and opposite effects on cardiovascular disease risk factors: the Quebec Family Study. Am J Clin Nutr 2001;74:315-21.

55 Després, JP; Prud'homme, D; Pouliot, MC; Tremblay, A; Bouchard, C. Estimation of deep abdominal adipose-tissue accumulation from simple anthropometric measurements in men. Am J Clin Nutr 1991;54:471-7.

56 Vague, J. The degree of masculine differentiation of obesities. Am J Clin Nutr 1956;4:2034.

57 Kannel, William B; Cupples, Adrienne; Ramaswami, Ratna; Stokes, Joseph III; Kreger, Bernard E. et al. Regional obesity and risk of cardiovascular diseasae; the Framingham Study. J Clin Epidemiol 1991;44(2):183-90.

58 Folsom, Aaron R; Kaye, Susan A; Sellers, Thomas A; Hong, Ching-Ping; Cerhan, James, $\mathrm{R}$. et al. Body fat distribution and 5-year risk of death in older women. JAMA 1993;269:483-87.

59 Rexrode, Kathrin M; Carey, Vincent J; Hennekens, Charles H; Walters, Ellen E; Colditz, Graham A et al. Abdominal adiposity and coronary heart disease in women. JAMA 1998;280:1843-48.

60 Gustat, Jeanette; Elkasabany, Abdalla; Srinivasan, Sathanur; Berenson, Gerald S. Relation of abdominal heigth to cardiovascular risk factors in young adults. The Bogalusa Heart Study. Am J Epidemiol 2000;151:885-91.

61 Björntorp, Per. Metabolic implications of body fat distribution. Diabetes Care 1991;14(12):1132-43.

62 Després J-P, Lemieux, S; Lamarche, B; Prud'homme, D; Moorjani, S. et al. The insulin resistance-dyslipidemic syndrome: contribution of visceral obesity and therapeutic implications. Intern J Obes 1995; 19(suppl 1):S76-S86.

63 Reaven Gerald. Metabolic syndrome; pathophysiology and implications for management of cardiovascular disease. Circulation 2002;106:286-88.

64 Heyward, Vivian H. e Stolarczyk, Lisa M. Avaliação da composição corporal aplicada. São Paulo, Manole, 2000.

65 Pouliot, Marie-Cristine; Després, Jean-Pierre; Lemieux, Simone; Moorjani, Sital; Bouchard, Claude; et al. Waist circunference and abdominal sagittal diameter: best simple anthropometric indexes of abdominal visceral adipose tissue accumulation and related cardiovascular risk in men and women. Am J Cardiol 1994;73:460-468.

66 Lean, M E J; Han, T S; Morrison, C E. Waist circunference as a measure for indicating need for weigth management. BMJ 1995;311:158-61.

67 Bray, GA. Pathophysiology of obesity. Am J Cin Nutr 1992;55:4885-87. 
68 Hauner, Hans; Stangl, Karl; Schmatz, Christian; Burger, Kurt; Blömer, Hans; Pfeiffer, Ernst-Friedrich. Body fat distribution in men with angiographically confirmed coronary artery disease. Atherosclerosis 1990;85:203-10.

69 Zamboni, Mauro; Armellini, Fabio; Sheiban, Imad; De Marchi, Margherita; Todesco, Tiziana et al. Relation of body distribution in men and degree of coronary narrowings in coronary artery disease. Am J Cardiol 1992;70:1135-38.

70 Hauner, H; Bognar, E; Blum, A . Body fat distribution and its association with metabolic and hormonal risk factors in women with angiographically assessed coronary artery disease. Evidence for the presence of a metabolic syndrome. Atherosclerosis 1994;105:209-16.

71 Hartz, Arthur; Grubb, Blair; Wild, Robert et al. The association of waist hip ratio and angiographically determined coronary artery disease. Intern J Obes 1990;14:657-65. 
5 ARTIGO EM PORTUGUÊS 


\title{
ASSOCIAÇÃO ENTRE A INGESTÃO DE MACRONUTRIENTES E A OBESIDADE ABDOMINAL COM A DOENÇA ARTERIAL CORONÁRIA
}

\author{
Magda Ambros Cammerer \\ Waldomiro Carlos Manfroi
}

Programa de Pós-graduação em Cardiologia e Ciências Cardiovasculares, Faculdade de Medicina da Universidade Federal do Rio Grande do Sul (UFRGS)

Endereço de correspondência:

Waldomiro Carlos Manfroi

Faculdade de Medicina da Universidade Federal do Rio Grande do Sul

Rua Ramiro Barcelos, 2350

Porto Alegre, RS, Brasil 90035-003

Fone: (51) 3316-5604/3316-5666

e-mail: wmanfroi@terra.com.br 


\title{
ASSOCIAÇÃO ENTRE A INGESTÃO DE MACRONUTRIENTES E A OBESIDADE ABDOMINAL COM A DOENÇA ARTERIAL CORONÁRIA
}

\author{
Magda Ambros Cammerer \\ Waldomiro Carlos Manfroi
}

\section{RESUMO}

Introdução: A incidência da doença arterial coronária é uma das principais causas de morbidade e motalidade em diversos países e o estudo dos fatores de risco têm grande importância na prevenção e no tratamento dessa enfermidade. Entre outros fatores, a obesidade e a obesidade abdominal têm sido associadas com a maior incidência de DAC. A ingestão diária de nutrientes também pode estar relacionada com essa doença, porém, uma vez que a alimentação é complexa e contém diversos nutrientes, ainda não foi possível elucidar o impacto da alimentação no risco de desenvolver a doença arterial coronária.

Objetivo: Avaliar a relação entre o consumo alimentar diário, a presença de obesidade abdominal e achados angiográficos de obstrução arterial em pacientes portadores de cardiopatia isquêmica, submetidos a cateterismo cardíaco.

Métodos: Foi realizado um estudo transversal, com 284 pacientes submetidos a cateterismo cardíaco, da unidade de hemodinâmica de um hospital universitário. Foi avaliada a RCQ, o IMC, a ingestão alimentar diária através de um inquérito nutricional, a análise bioquímica do sangue e a avaliação do laudo do cateterismo cardíaco.

Resultados: Dos pacientes avaliados, 172 indivíduos (60,6\%) apresentavam alterações em uma ou mais artérias coronárias. A ingestão média diária de calorias foi de $2450,56 \mathrm{Kcal} / \mathrm{dia}$. O consumo de proteínas foi em média $1,66 \mathrm{~g} / \mathrm{Kg} /$ dia, de carboidratos foi de $3,83 \mathrm{~g} / \mathrm{Kg} / \mathrm{dia}$ e de lipídeos foi de $1,21 \mathrm{~g} / \mathrm{Kg} /$ dia. A idade, o sexo masculino, os níveis séricos de triglicerídeos, o consumo de álcool e a glicemia em jejum foram estatisticamente significativos na análise multivariada.

Conclusão: Nos pacientes avaliados, o consumo diário de calorias encontra-se adequado, porém a ingestão de proteínas, carboidratos e lipídeos estão inadequados. Em relação aos fatores de risco para DAC, as mulheres apresentaram maior associação para desenvolver a síndrome metabólica do que os homens.

Palavras-chaves: inquéritos sobre dietas, relação cintura/quadril, fatores de risco, doença arterial coronária 


\section{INTRODUÇÃO}

Segundo estudos realizados na Europa, nos Estados Unidos e no Brasil, a incidência da doença arterial coronária (DAC) continua sendo a principal causa de morbidade e mortalidade em adultos. ${ }^{(1-3)}$

De acordo com dados do American Heart Association $(A H A)^{(4)}$, foram realizados avanços quanto ao entendimento da fisiopatologia da doença em relação às medidas de prevenção primária e secundária. Houve, também, um grande desenvolvimento na identificação de fatores causais e na prevenção dos mesmos para evitar os desfechos clínicos e/ou novos eventos, assim como a implementação de novas alternativas terapêuticas, tanto de ordem medicamentosa como procedimentos invasivos.

A etiologia da doença arterial coronária é complexa e multifatorial, segundo o AHA e o American College of Cardiology (ACC), os fatores de risco independentes para DAC são tabagismo, hipertensão arterial sistêmica (HAS), colesterol total elevado, lipoproteína de baixa densidade (LDL-C) elevada, lipoproteína de alta densidade (HDL-C) reduzida, diabete mellitus (DM) e idade avançada. ${ }^{(4)}$ Mais tarde, outros fatores foram associados com o aumento do risco para DAC denominando-se fatores de risco condicionantes e predisponentes.

A contribuição causal, determinante e quantitativa dos fatores de risco condicionantes ainda não foi bem documentada, porém, sabe-se que estão relacionados com o aumento do risco para DAC. São eles: triglicerídeos elevados, partículas LDL pequenas e densas, homocisteína sérica elevada, lipoproteína (a) sérica elevada, fatores protrombóticos (como fibrinogênio) e marcadores inflamatórios (como proteína C-reativa). ${ }^{(4)}$

Os fatores de risco predisponentes são aqueles que potencializam os fatores de risco independentes. São eles: obesidade ${ }^{(4,5)}$, obesidade abdominal $^{(6)}$, sedentarismo ${ }^{(7)}$, história familiar de DAC prematura em parentes de primeiro grau, características étnicas e fatores psicossociais. $^{(4)}$

Muitos destes fatores podem ser influenciadas por um ou mais componentes alimentares, entretanto, uma vez que a alimentação diária é complexa e contém diversos nutrientes e alimentos, ainda não foi possível elucidar ou quantificar precisamente o impacto específico da alimentação no risco de desenvolver doença arterial coronária. 
Informações associando os fatores da dieta com a incidência de doenças cardiovasculares originaram-se em estudos epidemiológicos, mostrando que populações com pouca incidência de cardiopatia isquêmica consumiam dietas pobres em gordura total, gordura saturada e colesterol; dietas ricas em fibras vegetais, e adequadas em relação às proteínas e aos carboidratos. $^{(9)}$

Desde os estudos de Framingham ${ }^{(10)}$, as evidências demonstram que a obesidade está associada também com os outros fatores de risco para DAC, como a hipertensão arterial sistêmica, colesterol total, HDL-colesterol, diabete mellitus, fatores protrombóticos e marcadores inflamatórios. ${ }^{(6,11)}$

Diversos estudos que analisaram a relação entre a distribuição da gordura corpórea "versus" o risco para DAC indicaram que a obesidade andróide (gordura na região abdominal) apresenta maior associação com a morbidade e a mortalidade do que a obesidade ginecóide (gordura na região glútea). ${ }^{(12)} \mathrm{A}$ idade, os hormônios sexuais, o sedentarismo, os fatores genéticos e dietéticos também podem induzir à obesidade abdominal. ${ }^{(13)}$

Assim, através deste estudo, pretende-se avaliar a associação entre os hábitos alimentares, a obesidade abdominal e a presença de doença arterial coronária, confirmada pela cinecoronariografia, em um grupo de pacientes do Hospital de Clínicas de Porto Alegre portadores de cardiopatia isquêmica.

\section{PACIENTES E MÉTODOS}

Foi realizado um estudo transversal com 284 pacientes (172 homens e 112 mulheres) da unidade de hemodinâmica do Hospital de Clínicas de Porto Alegre, com idade entre 29 e 85 anos, com o diagnóstico de angina estável, angina instável ou infarto do miocárdio, oriundos do ambulatório do HCPA ou encaminhados de outros serviços, no período de setembro de 2001 a junho de 2002. Foram excluídos do estudo os pacientes com insuficiência renal crônica, ascite, edema, acidente vascular cerebral, pacientes entubados ou em pós-operatório recente.

Do total de pacientes avaliados, 27 indivíduos (9,5\%) apresentavam angioplastia prévia com implante de "stent" e 19 pacientes $(6,7 \%)$ apresentavam cirurgia de revascularização do miocárdio. 
Antes de os pacientes serem submetidos à angiografia, foram esclarecidos da intenção do estudo proposto e convidados a participar do mesmo. Depois de concordarem em ingressar no estudo, assinavam um termo de consentimento (anexo), no qual autorizavam a realização de uma entrevista, avaliação antropométrica, coleta de $4 \mathrm{ml}$ de sangue para posterior análise bioquímica e avaliação do laudo do cateterismo cardíaco.

A avaliação antropométrica foi realizada após o paciente esvaziar a bexiga, vestindo roupas leves e sem sapatos. Foi utilizada balança de travessão com pesos móveis com aproximação de $100 \mathrm{~g}$, da marca $A$ rja ${ }^{\circledR}$. O paciente era posicionado na plataforma da balança, com o peso do corpo igualmente distribuído entre os pés, em ângulo reto. A precisão da balança era verificada periodicamente. A medida de altura em pé foi realizada com o paciente sob a plataforma da balança, com um estadiômetro com haste móvel. O paciente inspirava, enquanto o cursor horizontal do estadiômetro era baixado até o ponto mais alto da cabeça, comprimindo os cabelos. A altura em pé foi medida com aproximação de $0,1 \mathrm{~cm}$. As medidas de circunferências da cintura e do quadril foram feitas com fita métrica de metal, inelástica, da marca TBW, com intervalos de $0,1 \mathrm{~cm}$. A circunferência da cintura foi verificada no ponto médio entre a margem da costela inferior e a crista ilíaca, e a circunferência do quadril, no ponto mais largo ao redor dos trocânteres maiores. Foram feitas três medidas de circunferências e a média entre elas.

Os $4 \mathrm{ml}$ de sangue foram coletados através de introdutor arterial e colocados em frasco “BD Vacutainer ${ }^{\circledR}$ ", com gel separador. Uma hora após a coleta, o sangue era centrifugado durante 5 minutos a uma temperatura de $20^{\circ} \mathrm{C}$ e $3000 \mathrm{rpm}$, após o sangue era encaminhado ao laboratório de análises bioquímicas do HCPA onde foram realizadas as dosagens.

As entrevistas dos pacientes deram-se por intermédio de protocolo previamente testado (anexo). A idade e o sexo foram obtidos durante a entrevista, sendo que a idade foi calculada a partir da data de nascimento, até a data da entrevista, em anos completos.

O Índice de Massa Corpórea (IMC) foi obtido calculando o peso em quilogramas, dividido pela altura elevada ao quadrado. ${ }^{(14)}$ A relação cintura/quadril (RCQ) foi obtida pela divisão da circunferência da cintura em centímetros pela circunferência do quadril em centímetros. Os valores utilizados como pontos de corte para avaliar o risco através da circunferência abdominal nos homens foi $\geq 102 \mathrm{~cm}$ e nas mulheres foi $\geq 88 \mathrm{~cm}$. ${ }^{(15)}$ Para 
avaliar a relação cintura/quadril, foram utilizados os valores de $\geq 0,95$ para os homens $\mathrm{e} \geq$ 0,80 para as mulheres. ${ }^{(16)}$

As informações sobre diabete mellitus (DM) e hipertensão arterial sistêmica (HAS) foram obtidas durante a entrevista com o paciente e confirmadas através dos diagnósticos médicos, pela utilização de medicação específica e/ou dieta para o tratamento. Também foram coletadas informações a respeito do tempo de doença. A medicação em uso foi obtida pelo receituário médico fornecido pelo paciente.

A presença de dislipidemias foi questionada durante a entrevista com o paciente, e confirmados os diagnósticos através dos resultados dos exames laboratoriais.

Foi considerada história familiar positiva à existência de morte por infarto, cirurgia de revascularização do miocárdio e angioplastia em familiares como pai, mãe, irmãos e avós.

O sedentarismo foi analisado através da informação do paciente se este realizava atividade física semanal orientada. Foi considerado o tempo de realização da atividade física em: a) 1 a 3 horas por semana; b) 4 a 6 horas por semana; c) mais de 6 horas por semana.

A presença de tabagismo foi definida pelo consumo de mais de 5 cigarros por dia, pelo período de um ano ou mais. O consumo de álcool foi avaliado através do ingestão semanal de bebidas alcoólicas (cerveja, vinho ou destilados), considerando o volume que foi consumido em medidas caseiras e transformando em etanol $(1$ medida $=12 \mathrm{~g}$ etanol $)$.

O colesterol total foi medido pelo método CHOD-PAP, usando o equipamento Mega Bayer ${ }^{\circledR}$. O HDL-colesterol foi dosado pelo método direto por inibição seletiva, utilizando o equipamento Mega Bayer®. Os triglicerídeos foram avaliados pelo método GPO-PAP, usando equipamento Mega Bayer ${ }^{\circledR}$. O LDL-colesterol foi calculado através da equação de Friedewald. ${ }^{(17)}$ A glicose sérica foi avaliada pelo método Glico-DH, utilizando o equipamento Mega Bayer ${ }^{\circledR}$.

Foram considerados como risco para DAC, os valores de colesterol total $>200 \mathrm{mg} / \mathrm{dL}$ de sangue $^{(3)}$, para o HDL-colesterol $\leq 45 \mathrm{mg} / \mathrm{dL}$ para os homens e $\leq 55 \mathrm{mg} / \mathrm{dL}$ para as mulheres. ${ }^{(4)}$ A glicemia em jejum foi considerada $\geq 126 \mathrm{mg} / \mathrm{dL}^{(3)}$, para o LDL-colesterol foi considerado o valor $>130 \mathrm{mg} / \mathrm{dL}^{(3)}$ e para os triglicerídeos $>150 \mathrm{mg} / \mathrm{dL}$ de sangue. ${ }^{(3)}$ 
As informações a respeito da ingestão alimentar foram coletadas através de um inquérito recordatório de 24 horas, aplicado por nutricionista. O consumo de energia total foi calculado em calorias, utilizando o programa de computador Virtual Nutri da EPM/UNIFESP, versão $2.5^{(18)}$, cujos dados são compilações de várias tabelas de composição centesimal de alimentos nacionais e estrangeiras.

A análise de carboidratos, proteínas e gorduras foi realizada levando-se em consideração suas quantidades nas dietas, a porcentagem de calorias fornecidas por esses nutrientes em relação ao valor calórico total da dieta e a ingestão diária de cada macronutriente em grama por quilograma de peso do paciente. O padrão de adequação utilizado foi baseado nas recomendações da OMS. ${ }^{(19)}$

As informações sobre o comprometimento arterial foram indicadas através da análise da cinecoronáriografia, obtida pela técnica de Judkins. ${ }^{(20)} \mathrm{O}$ exame foi realizado na Unidade de Hemodinâmica do Serviço de Cardiologia do Hospital de Clínicas de Porto Alegre (HCPA). Foi definido como a existência de doença arterial coronariana a presença de estenose de $50 \%$ ou mais no diâmetro da coronária descendente anterior, coronária direita, circunflexa ou tronco. ${ }^{(21)}$ Também foi avaliado se o paciente fora submetido à cirurgia de revascularização do miocárdio ou angioplastia com "stent", ou sem uso de "stent" .

A análise estatística foi realizada com o auxílio do programa SPSS, versão 8.0, e STATA, versão 5.0. Os dados obtidos são apresentados sob a forma de proporções ou médias e desviopadrão. A avaliação da associação entre os possíveis fatores de risco e presença de lesões foi realizada através de teste $t$ de Student, para variáveis contínuas e do teste de qui-quadrado para variáveis categóricas. Os fatores significativos na análise bivariada foram estudados em conjunto através de uma regressão de Cox múltipla, conforme modelo proposto por Breslow, com estimativa robusta das variâncias. ${ }^{(22)}$ Foi escolhido este modelo estatístico, para testar a relação entre as variáveis independentes e a presença de achados angiográficos significativos, considerando a variável tempo=1 para todos os indivíduos. Desta forma, a medida de efeito calculada (hazard ratio) estima a razão de prevalências com um intervalo de confiança de 95\%. Em todas as comparações, foi considerado um alfa crítico de 0,05. 


\section{RESULTADOS}

\subsection{CARACTERÍSTICAS DA POPULAÇÃO}

A idade média dos pacientes avaliados foi de 59,1 \pm 11 anos, 60,6\% $(n=172)$ eram do sexo masculino, 63,4\% (n=180) apresentavam hipertensão arterial sistêmica e 57,7\% (n=164) eram tabagistas. Em relação à obstrução coronária, 172 pacientes $(60,6 \%)$ apresentaram alterações em uma ou mais artérias, sendo que a coronária descendente anterior foi a que apresentou maior número de obstruções significativas (45,4\%) (tab 1).

\subsection{ANÁLISE BIVARIADA}

A média de ingestão diária de calorias, na população, foi de 2450,56 Kcal/dia, com uma mediana $(2258,95 \mathrm{Kcal} / \mathrm{dia})$. A ingestão energética foi maior nos homens $(2658,72 \pm 982,71)$ do que nas mulheres $(2130,86 \pm 730,24 \mathrm{p}<0,001)$, assim como o consumo de quilocalorias por quilograma de peso por dia $(33,8 \pm 11,0$ versus $30,9 \pm 10,9 \mathrm{p}=0,03) .(\operatorname{tab} 4)$

Em relação à ingestão de macronutrientes, o consumo médio de proteínas foi de 1,66 \pm 0,67 g/Kg/dia, o consumo médio de carboidratos foi de 3,83 $\pm 1,45 \mathrm{~g} / \mathrm{Kg} / \mathrm{dia}$. Quanto à ingestão de lipídeos, a média foi de $1,21 \pm 0,58 \mathrm{~g} / \mathrm{Kg} /$ dia.

$\mathrm{Na}$ tabela 3, são apresentadas as médias e os desvios-padrões para os dois grupos de pacientes, com obstruções coronárias $(\mathrm{CAT}+)$ e sem obstruções coronárias significativas (CAT-). A relação cintura/quadril foi significativamente maior nos pacientes que tinham pelo menos uma artéria coronária com mais de 50\% de obstrução. A idade, a glicemia, o HDLcolesterol e triglicerídeos também foram estatisticamente significativos. No entanto, não foram encontradas diferenças em relação ao IMC e a circunferência abdominal nos dois grupos. O colesterol total e o LDL-colesterol, não apresentaram diferenças significativas, mesmo quando os pacientes relatavam fazer uso de medicação específica.

Quando analisados por sexo (tab 4), o valor médio do IMC nos homens foi de 27,7 $\pm 4,13$ $\mathrm{Kg} / \mathrm{m}^{2}$, e nas mulheres foi de $28,8 \pm 4,9 \mathrm{Kg} / \mathrm{m}^{2}$, indicando que a maior parte dos indivíduos 
deste estudo eram obesos. Isso resulta provavelmente de uma alta prevalência de sobrepeso e obesidade no Rio Grande do Sul ${ }^{(23)}$, região onde foi realizado o estudo.

A circunferência abdominal dos homens foi de $101,7 \pm 10,2 \mathrm{~cm}$, e nas mulheres foi de $97,1 \pm 11,6 \mathrm{~cm}(\mathrm{p}<0,001)$, mesmo após ajustado para os pontos de corte específicos para cada sexo. A relação cintura/quadril nos homens foi de $0,96 \pm 0,004$, enquanto, nas mulheres, esta relação foi de $0,91 \pm 0,05,(\mathrm{p}<0,001)$.

$\mathrm{Na}$ tabela de variáveis categóricas em relação ao sexo (tab 5), a hipertensão arterial sistêmica foi maior nas mulheres $(76,8 \%)$ do que nos homens $(54,7 \%)$, assim como, a história familiar positiva para DAC. A presença de tabagismo foi maior nos homens $(72,7 \%)$ do que nas mulheres $(34,8 \%)$, o consumo de álcool também foi superior nos homens.

\subsection{ANÁLISE MULTIVARIADA}

O escore angiográfico foi utilizado como variável dependente e os fatores de risco para doença arterial coronária (sexo masculino, idade, tabagismo, uso de álcool, diabete mellitus, hipertensão arterial sistêmica e atividade física), medidas antropométricas (índice de massa corpórea e relação cintura/quadril) e parâmetros bioquímicos (glicemia em jejum, HDLcolesterol, LDL-colesterol e triglicerídeos), como variáveis independentes para todos os indivíduos com obstrução coronária e sem obstrução coronária.

A idade, o sexo masculino, os níveis séricos de triglicerídeos, o consumo de álcool e a glicemia em jejum foram as variáveis estatisticamente significativas. Enquanto o HDLcolesterol, a relação cintura/quadril e o tabagismo não apresentaram significância estatística nesta amostra.

$\mathrm{Na}$ análise realizada separadamente para cada sexo, as mulheres apresentaram diferença estatística para o consumo de álcool $(\mathrm{p}=0,02)$, glicemia em jejum $(\mathrm{p}=0,015)$ e triglicerídeos $(p=0,023)$. Enquanto para os homens apenas a idade $(p=0,008)$ mostrou significância estatística. 


\section{DISCUSSÃO}

O presente estudo pretendeu avaliar a associação entre fatores da dieta e a obesidade abdominal, medida através da relação cintura/quadril, em pacientes portadores de cardiopatia isquêmica, submetidos a cateterismo cardíaco.

Destaca-se que os instrumentos de coleta de dados alimentares utilizados nesse trabalho não permitem avaliar criteriosamente a qualidade da alimentação dos pacientes, considerando, que foi realizado um inquérito recordatório de 24 horas. No entanto, foi escolhido este tipo de questionário, pois é fácil de ser aplicado, não altera a dieta usual, pode ser utilizado em grupos de baixa escolaridade e oferece uma boa estimativa do valor energético total da dieta e a ingestão de macronutrientes. ${ }^{(24)}$

O consumo médio de proteínas foi de $1,67 \mathrm{~g} / \mathrm{Kg} /$ dia, sendo que as recomendações diárias, segundo a OMS ${ }^{(25)}$, são em torno de 0,75 a 1 g/Kg/dia. Na avaliação da dieta, verificou-se que a população estudada consumia quase duas vezes mais proteínas do que as recomendações para indivíduos adultos.

Em estudos realizados em outros estados do Brasil ${ }^{(26)}$, o consumo de proteínas foi inferior ao encontrado na amostra estudada, no entanto, a população residente nesta região, culturalmente tem o hábito de ingerir proteínas de origem animal, principalmente provenientes da carne de gado, suíno e ovino. Estas fontes protéicas são ricas em ácidos graxos saturados, que contribuem para o aumento dos lipídeos séricos.

O consumo de carboidratos, foi quase a metade das recomendações segundo a OMS, que são de 6 a 7 g/Kg/dia. ${ }^{(19)}$ Apesar das fibras dietéticas não terem sido avaliadas separadamente neste estudo, os alimentos fontes de carboidratos complexos também são ricos em fibras solúveis, fibras insolúveis, vitaminas e minerais, sugerindo que a ingestão destes nutrientes por esta população, esteja abaixo das recomendações diárias. Estudos recentes demonstram que a alimentação rica em cereais ${ }^{(27)}$ e grãos integrais ${ }^{(28)}$ está associada à redução do risco de doenças cardiovasculares e distúrbios metabólicos.

A média diária de ingestão de lipídeos foi de $1,21 \mathrm{~g} / \mathrm{Kg} / \mathrm{dia}$, no entanto, as recomendações, são no máximo, de $1 \mathrm{~g} / \mathrm{Kg} / \mathrm{dia}$. Estudos realizados em diversas regiões do Brasil, avaliando o padrão alimentar da população urbana ${ }^{(29,30)}$, verificou-se um aumento significativo no consumo de gorduras na alimentação, assim como uma redução do consumo 
de carboidratos complexos e aumento de consumo de proteínas provenientes de carnes, leites e derivados. Demonstrando que a evolução alimentar do Brasil, passando de dietas hipocalóricas para normo ou hipercalóricas, ocorreu devido ao aumento da proporção de lipídeos na dieta. Fato este verificado em décadas passadas em países desenvolvidos e, mais recentemente, em países em desenvolvimento. ${ }^{(29,30)}$

A ingestão diária de calorias foi de 2450,56 Kcal/dia, sendo este valor muito próximo das recomendações para a população brasileira. ${ }^{(25)} \mathrm{O}$ consumo de calorias foi maior no grupo de pacientes que não apresentou obstruções significativas nas artérias coronárias. O que confirma a hipótese de que estes pacientes já recebem algum tipo de orientação nutricional. Porém, a adequação desta alimentação encontra-se comprometida pela ingestão elevada de proteínas e lipídeos, e ingestão reduzida de carboidratos.

$\mathrm{Na}$ amostra estudada, não houve correlação entre a ingestão diária de calorias e macronutrientes com as medidas das circunferências da cintura e do quadril, assim como com o índice de massa corpórea e a relação cintura/quadril. No entanto, estudos de base populacional realizados em outras regiões do Brasil confirmam estas evidências. ${ }^{(31)}$

Nesse estudo, na análise bivariada, verificou-se que a relação cintura/quadril foi significativamente maior nos indivíduos que apresentaram coronárias obstruídas do que nos indivíduos sem alterações coronarianas significativas. Porém, na análise multivariada, estes dados não são confirmados. Hauner e colaboradores ${ }^{(32,33)}$, ao realizarem uma análise semelhante avaliando homens e mulheres através de um estudo angiográfico, verificaram que apenas as mulheres acima de 60 anos apresentavam associação entre a RCQ com coronárias obstruídas. No presente estudo, não se estratificaram os indivíduos por faixas etárias e 39,4\% dos pacientes eram do sexo feminino, sugerindo que o tamanho da amostra avaliada não foi suficiente para encontrar as evidências de trabalhos anteriores.

$\mathrm{Na}$ análise multivariada, as mulheres apresentaram associação positiva para obstruções coronarianas, com a glicemia em jejum $(p=0,015)$ e triglicerídeos séricos $(p=0,023)$. Diversos estudos apresentam esta relação com a síndrome metabólica, associada à relação cintura/quadril alterada ${ }^{(5,13)}$, o que sugere, que as mudanças hormonais verificadas nas mulheres durante a menopausa modificam a distribuição de gordura, caracterizando este grupo com uma distribuição de gordura corporal "masculina", apresentando maior risco para diabete mellitus, intolerância à glicose e doenças do coração. ${ }^{(5)}$ 
Também foi verificada uma associação entre o sexo feminino, o consumo de álcool e a presença de coronárias obstruídas. Estudos populacionais realizados no Brasil, demonstraram uma associação entre a distribuição de gordura abdominal e a ingestão de bebidas destiladas em mulheres após a menopausa. ${ }^{(31)}$ Estes achados, porém, devem ser interpretados com cautela, pois a associação entre o consumo de álcool e a doença arterial coronariana continua controversa $^{(34)}$, e que a medida do consumo de álcool pode ser passível de erro, principalmente pelas mulheres que omitem mais freqüentemente informações a respeito do hábito de ingerir bebidas alcoólicas.

Como parte deste estudo, coletaram-se dados sobre outros fatores de risco para DAC, verificou-se que a glicemia em jejum, os triglicerídeos e o consumo de álcool foram associados com DAC em mulheres, mas não em homens. Nesta amostra, a idade elevada associada à DAC foi significativa para os homens, enquanto que o HDL-colesterol, a relação cintura/quadril e o tabagismo não foram significativos estatisticamente nos dois sexos. É importante ressaltar que as características da população estudada e o desenho do estudo, limitam a inferência sobre os fatores de risco para a população em geral.

Em relação ao tabagismo, um fator de risco independente e bem estabelecido para $\mathrm{DAC}^{(1,3,4)}$, neste estudo, não houve significância estatística, provavelmente pelo fato de os pacientes serem portadores de cardiopatia isquêmica e já terem sido orientados a parar de fumar. Hartz e colaboradores ${ }^{(35)}$, em um estudo semelhante, realizado com 272 pacientes submetidos a cateterismo cardíaco, também não encontraram associação entre tabagismo e doença arterial coronária.

Ao analisar os dados apresentados, verificou-se que os indivíduos avaliados apresentavam uma média de ingestão calórica diária dentro das recomendações da Organização Mundial da Saúde. No entanto, em relação à ingestão de macronutrientes (proteínas, carboidratos e lipídeos), encontrava-se inadequado, apresentando um elevado consumo de proteínas e lipídeos e baixo consumo de carboidratos.

Em relação às medidas antropométricas e o cateterismo, verificou-se que apenas a RCQ apresentou associação, porém, esta não foi confirmada quando corrigida para outros fatores. No entanto, a RCQ reflete não apenas as variações na distribuição da gordura visceral, mas, também, alterações da estrutura anatômica do quadril, como tamanho dos ossos da pelve, musculatura glútea e gordura subcutânea na região glútea. ${ }^{(36)}$ Quanto aos outros fatores de 
risco para DAC, as mulheres apresentam maior associação para desenvolver a síndrome metabólica do que os homens, confirmando dados de outros estudos. 


\section{REFERÊNCIAS}

1 Grundy, Scott M; Balady, Gary J; Criqui, Michael H e cols. Primary prevention of coronary heart disease: guidance from Framingham. A statement for healthcare professionals from the AHA task force on risk reduction. Circulation 1998;97;1876-87.

2 http://www.datasus.gov.br $(31 / 10 / 2002)$.

3 Grundy, SM; Pasternak, R; Greenland, P; et al. Assessment of cardiovascular risk by use of multiple-risk-factor assessment equations. A statement for healthcare professionals from the American Heart Association and the American College of Cardiology. Circulation 1999;100:1481-92.

4 Greenland, P; Grundy, S; Pasternak, MD; Lenfant, C. Problems on the pathway from risk assessment to risk reduction. Circulation 1998;97:1761-1762.

5 Grundy, Scott M. Obesity, metabolic syndrome, and coronary atherosclerosis. Circulation 2002;105:2696-98.

6 Eckel, RH.Obesity and heart disease; a statement for healthcare professionals from the nutrition committee, American Heart Association. Circulation 1997;96:3248-3250.

7 Eckel, Robert H; Barouch, Winifred W; Ershow, Abby G. Report of National Heart, Lung, and Blood Institute-National Institute of Diabetes and Digestive and Kidney Diseases Working Group on the Pathophysiology of Obesity-Associated Cardiovascular Disease. Circulation 2002;105:2923-28.

8 De Lorgeril, M; Salen, P; Martin, J-L, et al. Mediterranean diet, traditional risk factors, and the rate of cardiovascular complications after myocardial infarction: final report of the Lyon Diet Heart Study. Circulation 1999;99:779-85.

9 Mahan, Kathleen \& Escott-Stump, Sylvia. Alimentos, nutrição e dietoterapia. 9 ed. São Paulo, Roca, 1998.

10 Kannel, William; LeBauer, Joseph; Dawber, Thomas R; McNamara, Patricia. Relation of body weight to development of coronary heart disease. The Framingham Study. Circulation 1967 Apr;35(4):734-44.

11 Rosito, Guido Bernardo Aranha. Análise da associação entre níveis de fibrinogênio, fatores hemostáticos e redução do potencial fibrinolítico com o aumento da massa corporal e o acúmulo abdominal de gordura no Estudo dos Descendentes de Framingham, Tese (Doutorado) - Universidade Federal do Rio Grande do Sul.

12 Larsson, B; Svärdsudd, K; Welin, L; Wilhelmsen, L; Björntorp, P; Tibblin, G. Abdominal adipose tissue distribution, obesity, and risk of cardiovascular disease and death: 13 year follow up of participants in the study of men born in 1913. BMJ 1984;288:1401-04.

13 Björntorp, Per. Metabolic implications of body fat distribution. Diabetes Care 1991;14(12):1132-43. 
14 Garrow JS; Webster, J. Quetelet's index $\left(\mathrm{W} / \mathrm{H}^{2}\right)$ as a measure of fatness. Intern J Obes 1985;9:147-53.

15 Lean, M E J; Han, T S; Morrison, C E. Waist circunference as a measure for indicating need for weigth management. BMJ 1995;311:158-61.

16 Bray, GA. Pathophysiology of obesity. Am J Cin Nutr 1992;55:4885-87.

17 Friedewald, WT; Levy, R; Fredrickson, DS. Estimation of plasma low density lipoprotein cholesterol concentration without use of the preparative centrifuge. Cin Chem 1972;18:499.

18 Cis-EPM/Unifesp - Escola Paulista de Medicina. Sistema de apoio à decisão em nutrição - versão 2.5 .

19 GRUPO DE ESTUDIOS SOBRE DIETA, NUTRICIÓN Y PREVENCIÓN DE ENFERMIDADES NO TRANSMISSIBLES. Ginebra, 1989. Informe. Ginebra, Organización Mundial de la Salud, 1990. (OMS - Série Informes Técnicos, 797).

20 Judkins, MP. Selective coronary arteriography. I: A percuteneous transfemoral technique. Radiology 1967;89:815.

21 Chaitman, Bernard R; Bourassa, Martial G; Davis, Kathryn; Rogers, William J; Tyras, Denis $\mathrm{H}$, et al. Angiographic prevalence of high-risk coronary artery disease in patients subsets (CASS). Circulation 1981;64(2):360-7.

22 Lin, DY \& Wei, LJ. The robust interference for the Cox Porportionals Hazard Model. 1989;84:1074-8.

23 Gus, Iseu; Fischmann, Airton; Medina, Claúdio. Prevalência dos fatores de risco da doença arterial coronariana no Estado do Rio Grande do Sul. Arq Bras Cardiol 2002;78(5):478-83.

24 Cuppari, Lilian. Nutrição; nutrição clínica no adulto. Barueri, Manole, p.91, 2002.

25 REUNIÓN CONSULTIVA CONJUNTA FAO/OMS/UNU DE EXPERTOS EM NECESSIDADES DE ENERGIA Y PROTEÍNAS, Roma, 1981. Informe. Ginebra, Organización Mundial de la Salud, 1985. (OMS - Série de Informes Técnicos, 724).

26 Cervato, AM; Mazzilli, RN; Martins, IS; Marucci, MFN. Dieta habitual e fatores de risco para doenças cardiovasculares. Rev Saúde Pública 1997;31(3):227-35.

27 Truswell, AS. Cereal grains and coronary heart disease. Eur J Clin Nutr 2002;56(1):1-14.

28 McKeown, NM; Meigs, JB; Liu, S; Wilson, PW; Jacques, PF. Whole-grain intake is favorably associated wthi metabolic risk factors for type 2 diabetes and cardiovascular disease in the Framingham Offspring Study. Am J Clin Nutr 2002;76(2):390-8.

29 Mondini, Lenise e Monteiro, Carlos A. Mudanças no padrão de alimentação da população urbana brasileira (1962-1988). Rev Saúde Pública 1994;28(6):433-9. 
30 Monteiro, Carlos Augusto; Mondino, Lenise; Costa, Renata LB. Mudanças na composição e adequação nutricional da dieta familiar nas áreas metropolitanas do Brasil (1988-1996). Rev Saúde Pública 2000;34(3):251-8.

31 Machado, PAN; Sichieri, R. Relação cintura-quadril e fatores da dieta em adultos. Rev Saúde Pública 2002;36(2):198-204.

32 Hauner, Hans; Stangl, Karl; Schmatz, Christian; Burger, Kurt; Blömer, Hans; Pfeiffer, Ernst-Friedrich. Body fat distribution in men with angiographically confirmed coronary artery disease. Atherosclerosis 1990;85:203-10.

33 Hauner, H; Bognar, E; Blum, A . Body fat distribution and its association with metabolic and hormonal risk factors in women with angiographically assessed coronary artery disease. Evidence for the presence of a metabolic syndrome. Atherosclerosis 1994;105:209-16.

34 Lussier-Cacan, S; Bolduc, A; Xhignesse, M; Niyonsenga, T; Sing, CF. Impact of alcohol intake on measures of lipid metabolism depends on context defined by gender, body mass index, cigarette smoking, and apolipoprotein E genotype. Arterioscler Thromb Vasc Biol 2002;22(5):824-31.

35 Hartz, Arthur; Grubb, Blair; Wild, Robert et al. The association of waist hip ratio and angiographically determined coronary artery disease. Intern J Obes 1990;14:657-65.

36 Seidell, Jacob C; Pérusse, Louis; Després, Jean-Pierre; Bouchard, Claude. Waist and hip circumferences have independent and opposite effects on cardiovascular disease risk factors: the Quebec Family Study. Am J Clin Nutr 2001;74:315-21. 


\section{TABELAS}

Tabela 1

Descrição da população estudada quanto a sexo, fatores de risco e achados do cateterismo:

\begin{tabular}{|c|c|c|}
\hline Variáveis & $\mathrm{n}$ & $\%$ \\
\hline Sexo masculino & 172 & 60,6 \\
\hline Diabete mellitus 1 & 62 & 21,8 \\
\hline $\operatorname{HAS}^{1}$ & 180 & 63,4 \\
\hline Álcool & 107 & 37,7 \\
\hline Tabagismo & 164 & 57,7 \\
\hline Cateterismo +2 & 172 & 60,6 \\
\hline Coronária direita & 101 & 35,6 \\
\hline Circunflexa & 75 & 26,4 \\
\hline Descendente anterior & 129 & 45,4 \\
\hline Tronco & 5 & 1,8 \\
\hline "Stent" & 27 & 9,5 \\
\hline CRM & 19 & 6,7 \\
\hline
\end{tabular}

1 - presença da doença confirmada pelo uso de medicação ou dieta específicas, HAS, hipertensão arterial sistêmica.

${ }^{2}$ - presença de coronárias obstruídas no cateterismo; CRM, cirurgia de revascularização do miocárdio.

Tabela 2

Ingestão de nutrientes em pacientes com cateterismo normal e alterado:

\begin{tabular}{|c|c|c|c|}
\hline Variáveis & $\begin{array}{c}\text { CAT }+(n=172) \\
\text { Média } \pm \text { DP }\end{array}$ & $\begin{array}{c}\text { CAT }-(n=112) \\
\text { Média } \pm \text { DP }\end{array}$ & Sign. \\
\hline Kcal/dia & $2448,40 \pm 945,72$ & $2453,87 \pm 901,88$ & 0,96 \\
\hline $\mathrm{Kcal} / \mathrm{Kg} /$ dia & $32,27 \pm$ & $33,30 \pm 10,35$ & 0,44 \\
\hline Proteínas: $\mathrm{g} / \mathrm{Kg} / \mathrm{dia}$ & $1,66 \pm$ & $1,67 \pm$ & 0,89 \\
\hline Carboidratos: $\mathrm{g} / \mathrm{Kg} / \mathrm{dia}$ & $3,75 \pm$ & $3,94 \pm$ & 0,29 \\
\hline Lipídeos: $\mathrm{g} / \mathrm{Kg} / \mathrm{dia}$ & $1,18 \pm$ & $1,25 \pm$ & 0,31 \\
\hline
\end{tabular}


Tabela 3

Prevalência de fatores de risco para DAC em pacientes com cateterismo normal e alterado:

\begin{tabular}{lccl}
\hline \multicolumn{1}{c}{ Variáveis } & $\begin{array}{c}\text { CAT }+(\mathrm{n}=172) \\
\text { Média } \pm \mathrm{DP}\end{array}$ & $\begin{array}{c}\text { CAT }-(\mathrm{n}=112) \\
\text { Média } \pm \mathrm{DP}\end{array}$ & Sign. \\
& $60,33 \pm 11,04$ & $57,45 \pm 10,82$ & 0,031 \\
\hline Idade & $27,96 \pm 3,97$ & $28,48 \pm 5,22$ & 0,34 \\
IMC & $100,72 \pm 9,67$ & $98,70 \pm 12,42$ & 0,14 \\
Circ. Abdom. & $105,03 \pm 9,0$ & $105,31 \pm 11,02$ & 0,81 \\
Circ. quadril & $0,95 \pm 0,05$ & $0,93 \pm 0,06$ & 0,002 \\
RCQ & $101,49 \pm 43,40$ & $91,13 \pm 35,71$ & 0,035 \\
Glicemia em jejum & $202,19 \pm 51,41$ & $201,94 \pm 46,93$ & 0,97 \\
Colesterol total & $42,49 \pm 11,18$ & $46,22 \pm 12,57$ & 0,009 \\
HDL colesterol & $132,38 \pm 55,22$ & $130,80 \pm 46,55$ & 0,98 \\
LDL colesterol & $159,49 \pm 90,65$ & $138,16 \pm 67,87$ & 0,028 \\
Triglicerídeos & & & \\
\hline
\end{tabular}

Teste t de Student $(\alpha=0,05)$.

Tabela 4

Variáveis contínuas em relação ao sexo

\begin{tabular}{|c|c|c|c|c|c|}
\hline Variáveis & \multicolumn{2}{|c|}{$\begin{array}{c}\text { Homens } \\
\text { Média } \pm \text { DP }\end{array}$} & \multicolumn{2}{|c|}{$\begin{array}{c}\text { Mulheres } \\
\text { Média } \pm \text { DP }\end{array}$} & \multirow{2}{*}{$\frac{\text { Sign }}{0,23}$} \\
\hline Idade & 58,5 & $\pm 10,3$ & $60,1 \pm$ & 12,0 & \\
\hline IMC & 27,7 & $\pm \quad 4,13$ & 28,8 & 4,90 & 0,20 \\
\hline Circ. Abdom. & 101,7 & $\pm 10,2$ & 97,1 & 11,6 & $<0,001$ \\
\hline Circ. Quadril & 104,5 & $\pm \quad 9,0$ & $105,9 \pm$ & 10,9 & 0,26 \\
\hline $\mathrm{RCQ}$ & 0,96 & $\pm \quad 0,004$ & $0,91 \pm$ & 0,05 & $<0,001$ \\
\hline Glicemia & 95,8 & $\pm 39,2$ & $99,8 \pm$ & 43,1 & 0,43 \\
\hline Col. Total & 195,4 & $\pm 50,4$ & 212,2 & 46,6 & 0,005 \\
\hline HDL & 40,8 & $\pm 10,3$ & 48,7 & 12,4 & $<0,001$ \\
\hline LDL & 127,2 & $\pm 50,3$ & 138,0 & 53,5 & 0,07 \\
\hline Triglic & 152,3 & $\pm 84,3$ & $149,0 \pm$ & 81,1 & 0,74 \\
\hline $\mathrm{Kcal} / \mathrm{dia}$ & 2658,72 & $\pm 982,71$ & $2130,86 \pm$ & 730,24 & $<0,001$ \\
\hline $\mathrm{Kcal} / \mathrm{Kg} / \mathrm{dia}$ & 33,8 & $\pm 11,0$ & $30,9 \pm$ & 10,9 & 0,03 \\
\hline Proteínas: g/Kg/dia & 1,73 & $\pm \quad 0,67$ & $1,54 \pm$ & 0,63 & 0,01 \\
\hline Carboidratos: $\mathrm{g} / \mathrm{Kg} / \mathrm{dia}$ & 3,79 & 1,44 & $3,87 \pm$ & 1,47 & 0,64 \\
\hline Lipídeos: g/Kg/dia & 1,28 & 0,61 & $1,08 \pm$ & 0,51 & 0,004 \\
\hline
\end{tabular}

Qui-quadrado 
Tabela 5

Variáveis categóricas em relação ao sexo

\begin{tabular}{lrrr}
\hline \multicolumn{1}{c}{ Variáveis } & $\begin{array}{c}\text { Homens } \\
\mathrm{n}(\%)\end{array}$ & $\begin{array}{c}\text { Mulheres } \\
\mathrm{n}(\%)\end{array}$ & $\mathrm{p}$ \\
\hline Circ. Abdominal $^{1}$ & $79(46,2)$ & $92(53,8)$ & $<0,001$ \\
$\mathrm{RCQ}^{2}$ & $121(52,4)$ & $110(47,6)$ & $<0,001$ \\
$\mathrm{IMC}^{2}$ & & & \\
$\quad 25$ & $37(61,7)$ & $23(38,3)$ & 0,20 \\
$\quad 25-29,9$ & $87(64,4)$ & $48(35,6)$ & 0,20 \\
$\quad>30$ & $44(52,4)$ & $40(47,6)$ & 0,20 \\
$\mathrm{DM}$ & $39(22,7)$ & $23(20,5)$ & 0,77 \\
HAS & $94(54,7)$ & $86(76,8)$ & 0,002 \\
HFCPI & $103(59,9)$ & $82(73,2)$ & 0,029 \\
Tabagismo & $125(72,7)$ & $39(34,8)$ & $<0,001$ \\
Álcool & $84(48,8)$ & $23(20,5)$ & $<0,001$ \\
Atividade física & $111(64,5)$ & $81(72,3)$ & 0,48 \\
\hline
\end{tabular}

${ }^{1}$ homens $\geq 102 \mathrm{~cm}$, mulheres $\geq 88 \mathrm{~cm}$.

${ }^{2}$ homens $\geq 0,95$, mulheres $\geq 0,80$.

${ }^{3}$ história familiar de cardiopatia isquêmica qui-quadrado

Tabela 6

Análise de regressão de Cox:

\begin{tabular}{lccc}
\hline \multicolumn{1}{c}{ Variáveis } & significância & HR & IC95\% \\
\hline Sexo masculino & 0,013 & 1,39 & $1,07-1,79$ \\
Idade & 0,004 & 1,01 & $1,00-1,02$ \\
Tabagismo & 0,93 & 1,00 & $0,82-1,23$ \\
Consumo de álcool & 0,037 & 1,21 & $1,01-1,44$ \\
RCQ & 0,43 & 2,10 & $0,32-13,61$ \\
Glicemia em jejum & 0,051 & 1,00 & $0,99-1,00$ \\
HDL-colesterol & 0,19 & 1,00 & $0,98-1,00$ \\
Triglicerídeos & 0,027 & 1,00 & $1,00-1,00$ \\
\hline
\end{tabular}




\section{ANEXOS}

\section{TERMO DE CONSENTIMENTO INFORMADO}

Estamos realizando um estudo com pessoas que fazem exames de cinecoronáriografia no Hospital de Clínicas de Porto Alegre.

Este estudo, tem como objetivo, analisar a relação entre os hábitos alimentares e os fatores de risco para doenças cardíacas.

Para a análise do estudo, serão realizados os seguintes procedimentos:

- coleta de $10 \mathrm{ml}$ de sangue, para verificar o colesterol total, HDL-colesterol, LDLcolesterol, triglicerídios e glicemia.

- entrevista com o paciente feita pelo(a) pesquisador(a), para analisar o consumo diário de alimentos;

- avaliação do estado nutricional (verificar o peso, a altura, a circunferência da cintura e do quadril, medir a circunferência do braço e a dobra de gordura do braço).

Salientamos, que o procedimento não causará nenhum dano à saúde e o tratamento efetuado não será diferente se o(a) senhor(a) não concordar em participar de estudo, e que mesmo após ter assinado o termo poderá desistir do estudo à qualquer momento.

Informamos que se o(a) senhor(a) aceitar participar do estudo, não será identificado(a) e que se manterá o caráter confidencial de informações relacionadas com sua privacidade.

O pesquisador responsável por este estudo é o Dr. Waldomiro Carlos Manfroi (fones: 3328-8328 e 9982-0786) e a Nut ${ }^{\mathrm{a}}$ Magda Ambros Cammerer (fones: 3388-8548 e 9806-3749) é a executora deste projeto.

Se o(a) senhor(a) tiver alguma dúvida antes de decidir quanto a sua participação no estudo, sinta-se à vontade em perguntar.

Declaro diante das informações acima expostas, que estou de pleno acordo em participar da pesquisa.

Paciente:

Pesquisador responsável:
Responsável:

Data: 
PROTOCOLO DE ATENDIMENTO

Data:

Resultado do exame:
Protocolo $\mathrm{n}^{\circ}$ : $\mathrm{N}^{\circ}$ exame:

1. Identificação:

Prontuário:

Nome:

Idade: Data de nasc.:

Estado civil:

Sexo:(1)masc.(2)fem.

Profissão:

Endereço:

Cidade: CEP:

Telefone:

Nome do médico:

\section{Avaliação Nutricional}

Peso atual:

Peso usual:

Altura:

IMC:
Circunferência abdominal:

Circunferência do quadril:

Relação cintura/quadril:

\section{Medicação em Uso:}

\section{Avaliação do Estado Geral}

Mastigação:

Dentição:

Hábito intestinal

Uso de laxativos:

Hábito urinário:

Hidratação:

Alergias alimentares:

Quais:

Aversões alimentares:

Quais:

Apetite:
1 Normal

1 Completa

1 Regular

$1 \mathrm{Sim}$

1 Regular

1 Adequada

$1 \mathrm{Sim}$

$1 \mathrm{Sim}$

1 Bom
2 Prejudicada

2 Prejudicada

2 Irregular

2 Não

3 Eventualmente

2 Irregular

2 Inadequada

2 Não

2 Não

2 Regular

3 Inapetente 
Observações

adicionais:

\section{Fatores de Risco Referidos (marcar aqueles que o paciente refere)}

( ) DM Tempo de doença:

Tratada: 1 Sim 2 Não

( ) HAS Tempo de doença:

Tratada: 1 Sim 2 Não

( ) Dislipidemias

( ) Desconhecido

( ) Obesidade

( ) História familiar de CI: ( ) pais ( ) irmãos ( ) avós

Idade:
( ) Atividade física regular (horas/semana):
( ) História de tabagismo:
( ) Não ( ) 1-3
( ) $4-6$
( ) $>6$

Tempo de uso:

Cig/dia:

( ) Bebida alcoólica: quantidade/frequência:

\section{Exames bioquímicos}

Glicose:

Colesterol total:

HDL colesterol:

LDL colesterol:

Triglicerídeos:

\section{Anamnese alimentar:}

\begin{tabular}{|l|l|l|}
\hline \multicolumn{1}{|c|}{ REFEIÇÃO } & ALIMENTOS & QUANTIDADE \\
\hline CAFÉ DA MANHÃ & & \\
Horário: & & \\
\hline $\begin{array}{l}\text { LANCHE } \\
\text { Horário: }\end{array}$ & & \\
\hline $\begin{array}{l}\text { ALMOÇO } \\
\text { Horário: }\end{array}$ & & \\
& & \\
\hline LANCHE & & \\
Horário: & & \\
& & \\
\hline
\end{tabular}




\begin{tabular}{|l|l|l|}
\hline JANTAR & & \\
Horário: & & \\
\hline $\begin{array}{l}\text { CEIA } \\
\text { Horário: }\end{array}$ & & \\
\hline LANCHES NOS & & \\
INTERVALOS & & \\
& & \\
& & \\
\hline
\end{tabular}

Examinador:

Este trabalho foi realizado com financiamento do FIPE-HCPA. 
6 ARTIGO EM INGLÊS 
UNIVERSIDADE FEDERAL DO RIO GRANDE DO SUL

GRADUATE PROGRAM IN MEDICAL SCIENCES:

CARDIOLOGY AND CARDIOVASCULAR SCIENCES

MASTER'S DISSERTATION

ASSOCIATION OF MACRONUTRIENT INTAKE AND ABDOMINAL OBESITY WITH CORONARY HEART DISEASE

\author{
Magda Ambros Cammerer \\ Dissertation Advisor: Prof. Dr. Waldomiro Carlos Manfroi
}

Porto Alegre, November 2002 


\title{
ASSOCIATION OF MACRONUTRIENT INTAKE AND ABDOMINAL OBESITY WITH CORONARY HEART DISEASE
}

\author{
Magda Ambros Cammerer \\ Waldomiro Carlos Manfroi
}

\begin{abstract}
Introduction: the incidence of coronary heart disease (CHD) remains the main cause of morbidity and mortality among adults, and the study of risk factors is important in the prevention and treatment of this disease. Between other factors, obesity and abdominal obesity have been associated with CHD. Daily intake of nutrients can be related with this disease, however, daily diet is complex and contains several nutrients and foods, and the specific impact of diet on the risk of coronary heart disease has not yet been accurately explained or quantified .

Objective: to evaluate the relation between daily intake and abdominal obesity and angiography findings of coronary obstruction in patients with isquemic cardiopatic, submitted to a cardiac catheterization.

Methods: a cross study with 284 patients submitted a cardiac catheterization of the Hemodynamics Unit. It was evaluated the waist-rip-ratio, body mass index and daily intake.

Results: Coronary obstruction was observed in $172(60.6 \%)$ patients with abnormalities in one or more arteries. The analysis of macronutrient intake showed that mean protein intake was $1.66 \pm 0.65 \mathrm{~g} / \mathrm{Kg} /$ day, mean carbohydrate intake was $9.83 \pm 1.45 \mathrm{~g} / \mathrm{Kg} /$ day and mean lipid intake was $1.21 \pm 0.58 \mathrm{~g} / \mathrm{Kg} / \mathrm{day}$. Age, male sex, tryglicerides, alcohol intake and fasting glucose was statistically significant in multivariate analysis.

Conclusion: the energy intake is adjust, but the protein, carbohydrate and lipids are not. In relation to the others risk factors for CHD, the women were more significantly associated with the risk to develop metabolic syndrome than in men, which confirmed findings in other studies.
\end{abstract}

Key-words: daily intake, waist-rip-ratio, risk factors, cardiovascular disease. 


\section{INTRODUCTION}

Studies conducted in Europe, the United States and Brazil show that the incidence of coronary heart disease (CHD) remains the main cause of morbidity and mortality among adults. $^{(1-3)}$

Data from the American Heart Association (AHA) ${ }^{(4)}$, show advances in the understanding of the pathophysiology of this disease and the definition of primary and secondary preventive measures. Great advances in the identification of causal factors and their prevention have also been made, and new therapeutic alternatives - both drugs and invasive procedures - have been developed.

The etiology of coronary heart disease is complex and multifactorial. According to the AHA and the American College of Cardiology (ACC), independent risk factors for CHD are smoking, hypertension, elevated total cholesterol, elevated low-density lipoprotein cholesterol (LDL-C), reduced high density lipoprotein cholesterol (HDL-C), diabetes mellitus (DM) and advancing age. ${ }^{(4)}$ Lately, other factors have been associated with increased risk to develop $\mathrm{CHD}$, and risk factors were classified as conditional or predisposing.

The causative, independent and quantitative contribution of conditional risk factors have not been determined yet, but it is already known that these risk factors are associated with increased risk to develop CHD. Conditional risk factors are: elevated triglyceride concentrations, small dense LDL particles, elevated serum homocysteine concentrations, elevated serum lipoprotein (a) concentrations, prothrombotic factors (such as fibrinogen) and plasma markers for inflammation (such as C-reactive protein). ${ }^{(4)}$

Predisposing risk factors are those that worsen independent risk factors. They are: obesity $^{(4,5)}$, abdominal obesity ${ }^{(6)}$, and physical inactivity ${ }^{(7)}$, family history of premature CHD, ethnic caracteristics and phychosocial factors. ${ }^{(4)}$

Many of these factors may be worsened by one or more dietary components. However, daily diet is complex and contains several nutrients and foods, and the specific impact of diet on the risk of coronary heart disease has not yet been accurately explained or quantified .

Epidemiological studies have provided information about the association between diet and the incidence of cardiovascular disease, and have shown that populations with a low 
incidence of ischemic cardiopathy have diets that are low in total fats, saturated fats and cholesterol, rich in vegetable fibers, and balanced in terms of protein and carbohydrate intakes. ${ }^{(8)}$

Since the Framingham Heart Study ${ }^{(10)}$, evidence has shown that the association between obesity and cardiovascular disease is also influenced by other risk factors, such as hypertension, total cholesterol concentration, HDL-cholesterol, diabetes mellitus, prothrombotic factors, and markers for inflammation. ${ }^{(6,11)}$

Several studies analyzed the association between distribution of body fat and risk of CHD, and showed that android obesity (fat in the abdominal region) is more associated with morbidity and mortality than gynoid obesity (fat in the gluteal region). ${ }^{(12)}$ Age, sexual hormones, physical inactivity, genetic and dietary factors may also lead to abdominal obesity. $^{(13)}$

Therefore, the purpose of this study was to evaluate the association of dietary habits, abdominal obesity and coronary heart disease, confirmed by coronary cineangiography, in a group of patients of Hospital de Clínicas de Porto Alegre, Brazil, who had ischemic cardiopathy.

\section{PATIENTS AND METHODS}

It was done a cross section study and was conducted with 284 patients (172 men) of the Hemodynamics Unit, Hospital de Clínicas de Porto Alegre (HCPA), aged 29 to 85 years and with a diagnosis of stable angina, unstable angina, or myocardial infarction. Patients were referred to us by the outpatient service or by other services at HCPA from September 2001 to June 2002. We excluded from the study patients with chronic renal failure, ascites, edema, or cerebrovascular accident, and patients who were intubated or had recently undergone surgery.

Of all patients examined, $27(9.5 \%)$ had undergone previous angioplasty with stent implantation, and $19(6.7 \%)$ had undergone surgery for myocardial revascularization.

Patients were informed about the purposes of the study and invited to participate before they underwent angiography. After agreeing to participate, patients signed an informed consent document authorizing an interview, anthropometric evaluation, collection of a 4-mL 
blood sample for later biochemical analysis, and evaluation of cardiac catheterization findings.

Anthropometric evaluation was conducted with the patient barefoot, wearing light clothing, and after the patient had emptied the urinary bladder. We used an Arja ${ }^{\circledR}$ eye-level beam scale with movable weights and graduation in $100 \mathrm{~g}$ increments. Patient was placed on the scale platform at a right angle and with the weight of the body equally distributed between the feet. The scale was periodically calibrated. Height measurement was performed with a stadiometer with a movable height rod. The patient stood on the scale platform, and inhaled while the headpiece of the stadiometer was lowered to the highest point on the top of the head, pushing down the hair. Standing height was measured to the nearest $0.1 \mathrm{~cm}$. Waist and hip circumferences were measured with an inelastic metal measuring tape (TBW) with $0.1 \mathrm{~cm}$ graduation. Waist circumference was measured at the midpoint between the lowest rib and the iliac crest, and hip circumference was measured at the widest hip area, usually around the greater trochanters. We collected three measurements of the circumferences and calculated their averages.

A 4-mL arterial blood sample was collected and placed in a BD Vacutainer ${ }^{\circledR}$ container with separating gel. One hour after collection blood was centrifuged for 5 minutes at $20^{\circ} \mathrm{C}$ and $3000 \mathrm{rpm}$. The sample was then sent to the biochemical analysis laboratory at HCPA where concentrations were measured.

Patients were interviewed according to a previously tested protocol. Data about age and sex were obtained during the interview: age was calculated as full years from the date of birth to the date of the interview.

Body mass index was calculated by dividing weight in kilograms by height in square centimeters. ${ }^{(14)}$ The waist-to-hip ratio was obtained by dividing waist circumference by hip circumference. Cutoff points for the evaluation of abdominal circumference were $\geq 102 \mathrm{~cm}$ for men and $\geq 88 \mathrm{~cm}$ for women. ${ }^{(15)}$ To evaluate waist-to-hip ratio we used the values of $\geq$ 0.95 for men and $\geq 0.80$ for women. ${ }^{(16)}$

Data about diabetes mellitus (DM) and hypertension were collected during the interview with the patient, and confirmed by medical diagnosis evidenced by specific medication and/or diet followed. Data about the duration of disease were also collected. The medication being used was confirmed by checking the prescription provided by the patient. 
Patients were asked about dyslipidemia during the interview, and diagnoses were confirmed by the results of laboratory tests.

Patients had a positive family history if they reported death due to infarction, myocardial revascularization surgery, or angioplasty of family members such as father, mother, siblings and grandparents.

Physical inactivity was evaluated by means of patients reports about supervised weekly physical activities. Physical activity was classified in three different groups:

(a) 1 to 3 hours per week; (b) 4 to 6 hours per week; (c) more than 6 hours per week.

Smoking was defined as more than 5 cigarettes per day for one year or longer. Alcohol consumption was calculated according to the weekly consumption of alcoholic beverages (beer, wine, liquor); domestic measures were converted into amounts of ethanol $(1$ measure $=$ $12 \mathrm{~g}$ ethanol).

Total cholesterol was measured by means of the CHOD-PAP method with a Mega Bayer ${ }^{\circledR}$ analyzer. HDL-cholesterol was measured with a direct assay by selective inhibition using a Mega Bayer ${ }^{\circledR}$ analyzer. Triglycerides were evaluated by the GPO-PA method, using the Mega Bayer ${ }^{\circledR}$ analyzer. LDL-cholesterol was calculated according to the equation proposed by Friedewald and colleagues. ${ }^{(17)}$ Serum glucose was measured by means of the Glyco-DH method using a Mega Bayer® analyzer.

Cutoff points adopted were: total cholesterol $>200 \mathrm{mg} / \mathrm{dL}{ }^{(1)}$, HDL-cholesterol $\leq 45$ $\mathrm{mg} / \mathrm{dL}$ for men and $\leq 55 \mathrm{mg} / \mathrm{dL}$ for women. ${ }^{(3)}$ Cutoff point for fasting glucose was $\geq 126$ $\mathrm{mg} / \mathrm{dL}^{(1)}$, for LDL-cholesterol, $>130 \mathrm{mg} / \mathrm{dL}$ (1), and for triglycerides, $>150 \mathrm{mg} / \mathrm{dL} .{ }^{(1)}$

Data about dietary intake were collected by means of a 24-hour recall questionnaire applied by a nutritionist. Total energy consumption was calculated in calories using the EPM/UNIFESP's software Virtual Nutri $2.5^{(18)}$ whose data are compilations of several tables of composition of national and foreign foods.

The analysis of carbohydrates, proteins and fats was performed according to their amounts in diets, the percentage of calories provided by these nutrients in relation to total dietary energy intake, and the daily intake of each macronutrient in grams per kilogram of the patient's weight. Daily allowances adopted were the ones recommended by the WHO. ${ }^{(19)}$ 
Data about arterial obstruction were obtained by means of the analysis of coronary cineangiography performed according to the Judkins technique. ${ }^{(20)}$ Examinations were performed in the Hemodynamics Unit of the Cardiology Service, HCPA. The existence of coronary heart disease was defined by the presence of stenosis of $50 \%$ or more of the diameter of the anterior descending, right, circumflex or trunk coronary. ${ }^{(21)} \mathrm{We}$ also collected data about whether the patient had undergone myocardial revascularization or angioplasty, with or without the use of stent.

Statistical analysis was performed with the softwares SPSS 8.0 and STATA 5.0. Data are presented as percentages or means and standard deviation. The evaluation of the association between risk factors and lesions was performed with the use of the Student $t$ test for continuous variables, and the chi-square test for categorical variables. Significant factors in bivariate analysis were studied as a whole by means of a multivariate Cox regression model according to the model proposed by Breslow, with robust estimation of variances. ${ }^{(22)} \mathrm{We}$ chose this model, to test the relationship between independent variables and significant angiographic findings, taking the variable "time" $=1$ for all individuals. These way, the Hazard Ratio apraised the ratio of prevalence wiht 95\% confidence interval. Significance for all comparisons was established at $\alpha=0.05$.

\section{RESULTS}

\subsection{CHARACTERISTICS OF THE POPULATION}

The mean age was $59,1 \pm 11$ years, $60.6 \%(n=172)$ were men, $63.4 \%(n=180)$ had hypertension, and $57.5 \%(\mathrm{n}=164)$ were smokers. Coronary obstruction was observed in 172 $(60.6 \%)$ patients with abnormalities in one or more arteries; the anterior descending coronary was the vessel that had the largest number of significant obstructions (45.4\%). (table 1).

\subsection{BIVARIATE ANALYSIS}

Mean daily energy intake was $2450.56 \mathrm{Kcal} /$ day; median was $2258.95 \mathrm{Kcal} /$ day. Energy intake was higher for men $(2658.72 \pm 982.71)$ than for women $(2130.86 \pm 730.24 ; \mathrm{p}<0.001)$; 
daily intake of kilocalories per kilogram of weight was also higher for men $(33.8 \pm 11.0$ versus $30.9 \pm 10.9 ; \mathrm{p}=0.03)$.(table 4 )

The analysis of macronutrient intake showed that mean protein intake was $1.66 \pm 0.65$ $\mathrm{g} / \mathrm{Kg} /$ day. and mean carbohydrate intake was $9.83 \pm 1.45 \mathrm{~g} / \mathrm{Kg} /$ day. Mean lipid intake was $1.21 \pm 0.58 \mathrm{~g} / \mathrm{Kg} /$ day.

Table 3 shows means and standard deviations for patients with coronary obstruction $(\mathrm{CAT}+)$ and without coronary obstruction (CAT-). The waist-to-hip ratio was significantly greater in patients that had at least one artery with more than $50 \%$ obstruction. However, no significant differences were found in BMI and abdominal circumference between the two groups. Age, fasting glucose, HDL-cholesterol and triglycerides were also significantly different. Total cholesterol and LDL-cholesterol were not significantly different, not even in patients who reported making use of specific medication.

Mean body mass indices were $27.7 \pm 4.13 \mathrm{Kg} / \mathrm{m}^{2}$ for men and $28.8 \pm 4.12 \mathrm{Kg} / \mathrm{m}^{2}$ for women, which shows that most of the subjects in this study were obese. This may reflect the high prevalence of overweight in the State of Rio Grande do Sul ${ }^{(23)}$ where the study was conducted.

Abdominal circumference was $101.7 \pm 10.2 \mathrm{~cm}$ in men and $97.1 \pm 11.6 \mathrm{~cm}$ in women $(\mathrm{p}<0.001)$ after adjustment to the cutoff points for each sex. The waist-to-hip ratio was $0.96 \pm$ $0.004 \mathrm{~cm}$ for men, and $0.91 \pm 0.05 \mathrm{~cm}$ for women $(\mathrm{p}<0.001)$.

The table where categorical variables according to sex are presented shows that hypertension was more frequent among women (76.8\%) than among men (54.7\%). A positive family history of CHD was also more frequent among women than among men. Smoking was more frequent among men (72.7\%) than among women (34.8\%). Alcohol consumption was also more frequent among men (48.4\%) (table 5).

\subsection{MULTIVARIATE ANALYSIS}

The angiographic score was the dependent variable, while independent variables for individuals with or without coronary obstruction were the risk factors for coronary heart disease (male, age, smoking, alcohol ingestion, diabetes, hypertension, physical activity), 
anthropometric measurements (body mass index and waist-to-hip ratio) and biochemical parameters (fasting glucose, HDL-cholesterol, LDL-cholesterol and triglycerides).(table 6)

When groups were separated according to sex, women showed significant differences for alcohol ingestion $(p=0.02)$. fasting glucose $(p=0.015)$ and triglyceride $(p=0.023)$. For men, only age $(p=0.008)$ was statistically significant.

\section{DISCUSSION}

This study evaluated the relationship between dietary factors and abdominal obesity measured by means of the waist-to-hip ratio in patients with ischemic cardiopathy who underwent cardiac catheterization.

It is important to point out that the instrument for dietary data collection used in this study - a 24-hour recall questionnaire - does not warrant an accurate evaluation of the amount of foods in the patients' diets. However, we chose this questionnaire because it is easy to apply, does not change usual diet, may be applied to subjects with little schooling, and provides a good estimate of the total dietary energy intake and macronutrient intake ${ }^{(24)}$.

The analysis of macronutrient intake showed that mean protein intake was $1.66 \pm 0.67$ $\mathrm{g} / \mathrm{Kg} / \mathrm{day}$, whereas the daily allowance recommended by the $\mathrm{WHO}{ }^{(25)}$ ranges from 0.75 to 1 $\mathrm{g} / \mathrm{Kg} / \mathrm{day}$. Diet analysis revealed that the intake for this population was more than twice the daily allowance for adults.

Studies conducted in other regions of Brazil ${ }^{(26)}$ revealed mean protein intake lower that that found in our study. However, the population living in this region, have the custom of protein intake is high, particularly when proteins are of animal origin - like cattle, swine, ovine - and found in foods that also contain lipids in their composition, are more likely to ingest saturated fats than individuals whose protein intake conforms to daily allowances.

Mean carbohydrate intake was $3.83 \pm 1.45 \mathrm{~g} / \mathrm{Kg} /$ day, about half the daily allowance recommended by the WHO, which ranges from 6 to $7 \mathrm{~g} / \mathrm{Kg} / \mathrm{day} .{ }^{(19)}$ Complex carbohydrate foods are also rich in soluble and insoluble fibers. Although dietary fibers were not separately measured in our study, low carbohydrate intake suggests that our patients' food fiber intake is below the recommended daily allowance. Recent studies reported that a diet rich in cereals ${ }^{(27)}$ 
and whole grains ${ }^{(28)}$ is associated with a reduction in the risk of cardiovascular disease and metabolic disorders.

Mean daily lipid intake was $1.21 \pm 0.58 \mathrm{~g} / \mathrm{Kg} /$ day; the $\mathrm{WHO}{ }^{(19)}$ daily allowance is 1 $\mathrm{g} / \mathrm{Kg} /$ day. Studies carried out in several regions in Brazil evaluated the diet patterns of urban populations ${ }^{(29,30)}$, and revealed a significant increase in fat intake in the diet of the general population, as well as a reduction in complex carbohydrate intake and increase in the ingestion of proteins from meat, milk and dairy products. These findings are evidence of the evolution of diet in Brazil, which went from hypocaloric diets to normal or hypercaloric diets in consequence of an increase in the proportion of diet lipids. This same fact was observed in developed countries in the past, and, more recently, in developing countries. ${ }^{(29,30)}$

Daily energy intake $(2453.87 \pm 901.88 \mathrm{Kcal} /$ day, value very close to the daily allowance recommended for the Brazilian population. Calories intake was higher in group of patients without coronary obstruction. This confirms the hypothesis that these patients had already received some nutritional supervision, but the adequacy of this diet is compromised by high protein and lipid intakes and a low carbohydrate intake.

In our series, there was no correlation between the daily intake of calories and macronutrients and the measurements of waist and hip circumferences, BMI and WHR. Population-based studies carried out in other regions of Brazil confirm this evidence. ${ }^{(31)}$

Bivariate analysis of the measurements of circumferences and their association with obstructed coronaries revealed that the waist-to-hip ratio was significantly greater in individuals who had obstructed coronaries than in individuals without significant coronary obstruction. Multivariate analysis, however, did not confirm these findings. Hauner and colleagues ${ }^{(32,33)}$ conducted a similar study to evaluate men and women by means of angiographic studies, and reported that the association of WHR with obstructed coronaries was only observed for women older than 60 years. In our study, we did not stratify data according to age groups, and $39.4 \%$ of the patients were women, which suggests that the sample size was not large enough to produce the evidence reported in previous studies.

Multivariate analysis revealed a positive association of coronary obstruction, fasting glucose $(p=0.015)$ and serum triglycerides $(p=0.023)$ in women. Several studies have reported this relationship to the metabolic syndrome in association with the waist-to-hip ratio ${ }^{(5,13)}$, which suggests that hormonal changes observed in women during menopause change the 
distribution of fat and give this group of women a "male" distribution of body fat. Therefore, they are at increased risk to develop diabetes mellitus, intolerance to glucose and heart disease, which characterize the metabolic syndrome ${ }^{(5)}$.

It was also an association between female sex, alcohol consumption and obstructed arteries in a multivariate analysis $(\mathrm{p}=0.020)$. Population-based studies in Brazil revealed the association between the distribution of abdominal fat and the ingestion of liquor in postmenopausal women. ${ }^{(31)}$ However, this informantions must be seen with caution bacause the association between alcohol consumption and coronary heart disease is still controversial (34), and that the measurement of amounts of alcohol consumption may be underestimated, especially among women, who more often omit information about the habit of consuming alcoholic beverages.

As part of our study, data about other risk factors for CHD were collected. We observed that fasting glucose, triglycerides and alcohol consumption are associated with CHD in women, but not in men. Advancing age associated with CAD was statistically significant for men, while HDL-cholesterol, waist-to-hip ratio and smoking were significant for both sexes. It is important to distinguish that the population's caracteristics and the design of the study restrict the inference about risk factors in general population.

Smoking is a well-established independent risk factor of CHD. ${ }^{(1,3,4)}$ In our study, it did not reach statistical significance, maybe because patients had ischemic cardiopathy and had already been advised to stop smoking. Hartz and colleagues ${ }^{(35)}$ carried out a similar study with 272 patients who underwent cardiac catheterization, and also did not find any association between smoking and coronary heart disease. Another fact worth mentioning is that our patients are sick individuals, and the other risk factors for CHD may weaken the association between smoking and coronary heart disease as determined by angiography.

The analysis of data in this study revealed that the patients evaluated had a mean daily calorie intake that conformed to the WHO recommended daily allowance. However, macronutrient intake (proteins, carbohydrates and lipids) was inadequate, with high protein and lipid intake and low carbohydrate intake.

The analysis of anthropometric measurements and catheterization findings revealed that only WHR was significantly associated with risk, but this association was not confirmed when adjusted to other factors. However, WHR reflects not only the variations in visceral fat 
distribution, but also the changes in the anatomic structure of the hip, such as the size of pelvic bones, gluteal muscles, and subcutaneous fat in the gluteal region. ${ }^{(36)}$ The analysis of other risk factors for CHD showed that they were more significantly associated with the risk to develop metabolic syndrome in women than in men, which confirmed findings in other studies. 


\section{REFERENCES}

1 Grundy, Scott M; Balady, Gary J; Criqui, Michael H e cols. Primary prevention of coronary heart disease: guidance from Framingham. A statement for healthcare professionals from the AHA task force on risk reduction. Circulation 1998;97;1876-87.

2 http://www.datasus.gov.br $(31 / 10 / 2002)$.

3 Grundy, SM; Pasternak, R; Greenland, P; et al. Assessment of cardiovascular risk by use of multiple-risk-factor assessment equations. A statement for healthcare professionals from the American Heart Association and the American College of Cardiology. Circulation 1999;100:1481-92.

4 Greenland, P; Grundy, S; Pasternak, MD; Lenfant, C. Problems on the pathway from risk assessment to risk reduction. Circulation 1998;97:1761-1762.

5 Grundy, Scott M. Obesity, metabolic syndrome, and coronary atherosclerosis. Circulation 2002;105:2696-98.

6 Eckel, RH.Obesity and heart disease; a statement for healthcare professionals from the nutrition committee, American Heart Association. Circulation 1997;96:3248-3250.

7 Eckel, Robert H; Barouch, Winifred W; Ershow, Abby G. Report of National Heart, Lung, and Blood Institute-National Institute of Diabetes and Digestive and Kidney Diseases Working Group on the Pathophysiology of Obesity-Associated Cardiovascular Disease. Circulation 2002;105:2923-28.

8 De Lorgeril, M; Salen, P; Martin, J-L, et al. Mediterranean diet, traditional risk factors, and the rate of cardiovascular complications after myocardial infarction: final report of the Lyon Diet Heart Study. Circulation 1999;99:779-85.

9 Mahan, Kathleen \& Escott-Stump, Sylvia. Alimentos, nutrição e dietoterapia. 9 ed. São Paulo, Roca, 1998.

10 Kannel, William; LeBauer, Joseph; Dawber, Thomas R; McNamara, Patricia. Relation of body weight to development of coronary heart disease. The Framingham Study. Circulation 1967 Apr;35(4):734-44.

11 Rosito, Guido Bernardo Aranha. Análise da associação entre níveis de fibrinogênio, fatores hemostáticos e redução do potencial fibrinolítico com o aumento da massa corporal e o acúmulo abdominal de gordura no Estudo dos Descendentes de Framingham, Tese (Doutorado) - Universidade Federal do Rio Grande do Sul.

12 Larsson, B; Svärdsudd, K; Welin, L; Wilhelmsen, L; Björntorp, P; Tibblin, G. Abdominal adipose tissue distribution, obesity, and risk of cardiovascular disease and death: 13 year follow up of participants in the study of men born in 1913. BMJ 1984;288:1401-04.

13 Björntorp, Per. Metabolic implications of body fat distribution. Diabetes Care 1991;14(12):1132-43. 
14 Garrow JS; Webster, J. Quetelet's index $\left(\mathrm{W} / \mathrm{H}^{2}\right)$ as a measure of fatness. Intern J Obes 1985;9:147-53.

15 Lean, M E J; Han, T S; Morrison, C E. Waist circunference as a measure for indicating need for weigth management. BMJ 1995;311:158-61.

16 Bray, GA. Pathophysiology of obesity. Am J Cin Nutr 1992;55:4885-87.

17 Friedewald, WT; Levy, R; Fredrickson, DS. Estimation of plasma low density lipoprotein cholesterol concentration without use of the preparative centrifuge. Cin Chem 1972;18:499.

18 Cis-EPM/Unifesp - Escola Paulista de Medicina. Sistema de apoio à decisão em nutrição - versão 2.5 .

19 GRUPO DE ESTUDIOS SOBRE DIETA, NUTRICIÓN Y PREVENCIÓN DE ENFERMIDADES NO TRANSMISSIBLES. Ginebra, 1989. Informe. Ginebra, Organización Mundial de la Salud, 1990. (OMS - Série Informes Técnicos, 797).

20 Judkins, MP. Selective coronary arteriography. I: A percuteneous transfemoral technique. Radiology 1967;89:815.

21 Chaitman, Bernard R; Bourassa, Martial G; Davis, Kathryn; Rogers, William J; Tyras, Denis $\mathrm{H}$, et al. Angiographic prevalence of high-risk coronary artery disease in patients subsets (CASS). Circulation 1981;64(2):360-7.

22 Lin, DY \& Wei, LJ. The robust interference for the Cox Porportionals Hazard Model. 1989;84:1074-8.

23 Gus, Iseu; Fischmann, Airton; Medina, Claúdio. Prevalência dos fatores de risco da doença arterial coronariana no Estado do Rio Grande do Sul. Arq Bras Cardiol 2002;78(5):478-83.

24 Cuppari, Lilian. Nutrição; nutrição clínica no adulto. Barueri, Manole, p.91, 2002.

25 REUNIÓN CONSULTIVA CONJUNTA FAO/OMS/UNU DE EXPERTOS EM NECESSIDADES DE ENERGIA Y PROTEÍNAS, Roma, 1981. Informe. Ginebra, Organización Mundial de la Salud, 1985. (OMS - Série de Informes Técnicos, 724).

26 Cervato, AM; Mazzilli, RN; Martins, IS; Marucci, MFN. Dieta habitual e fatores de risco para doenças cardiovasculares. Rev Saúde Pública 1997;31(3):227-35.

27 Truswell, AS. Cereal grains and coronary heart disease. Eur J Clin Nutr 2002;56(1):1-14.

28 McKeown, NM; Meigs, JB; Liu, S; Wilson, PW; Jacques, PF. Whole-grain intake is favorably associated wthi metabolic risk factors for type 2 diabetes and cardiovascular disease in the Framingham Offspring Study. Am J Clin Nutr 2002;76(2):390-8.

29 Mondini, Lenise e Monteiro, Carlos A. Mudanças no padrão de alimentação da população urbana brasileira (1962-1988). Rev Saúde Pública 1994;28(6):433-9. 
30 Monteiro, Carlos Augusto; Mondino, Lenise; Costa, Renata LB. Mudanças na composição e adequação nutricional da dieta familiar nas áreas metropolitanas do Brasil (1988-1996). Rev Saúde Pública 2000;34(3):251-8.

31 Machado, PAN; Sichieri, R. Relação cintura-quadril e fatores da dieta em adultos. Rev Saúde Pública 2002;36(2):198-204.

32 Hauner, Hans; Stangl, Karl; Schmatz, Christian; Burger, Kurt; Blömer, Hans; Pfeiffer, Ernst-Friedrich. Body fat distribution in men with angiographically confirmed coronary artery disease. Atherosclerosis 1990;85:203-10.

33 Hauner, H; Bognar, E; Blum, A . Body fat distribution and its association with metabolic and hormonal risk factors in women with angiographically assessed coronary artery disease. Evidence for the presence of a metabolic syndrome. Atherosclerosis 1994;105:209-16.

34 Lussier-Cacan, S; Bolduc, A; Xhignesse, M; Niyonsenga, T; Sing, CF. Impact of alcohol intake on measures of lipid metabolism depends on context defined by gender, body mass index, cigarette smoking, and apolipoprotein E genotype. Arterioscler Thromb Vasc Biol 2002;22(5):824-31.

35 Hartz, Arthur; Grubb, Blair; Wild, Robert et al. The association of waist hip ratio and angiographically determined coronary artery disease. Intern J Obes 1990;14:657-65.

36 Seidell, Jacob C; Pérusse, Louis; Després, Jean-Pierre; Bouchard, Claude. Waist and hip circumferences have independent and opposite effects on cardiovascular disease risk factors: the Quebec Family Study. Am J Clin Nutr 2001;74:315-21. 


\section{TABLES}

Table 1

Characteristics of the study population about sex, risk factors and angiography:

\begin{tabular}{lrr}
\hline Variables & $\mathrm{N}$ & $\%$ \\
\hline Male & 172 & 60.6 \\
Diabetes mellitus & 62 & 21.8 \\
$\mathrm{H}^{1}$ & 180 & 63.4 \\
Alcohol & 107 & 37.7 \\
Smoking & 164 & 57.7 \\
Catheterization + & 172 & 60.6 \\
$\quad$ Right coronary & 101 & 35.6 \\
$\quad$ Circumflex & 75 & 26.4 \\
$\quad$ Anterior ascending & 129 & 45.4 \\
$\quad$ Trunk & 5 & 1.8 \\
$\quad$ Stent & 27 & 9.5 \\
$\quad$ MRS & 19 & 6.7 \\
\hline
\end{tabular}

1 - disease confirmed by use of specific medication or diet; $\mathrm{H}$ - hypertension.

${ }^{2}$ - obstructed coronaries at catheterization; MRS - myocardial revascularization surgery.

Table 2

Nutrient intake and patients with normal and changed catheterization:

\begin{tabular}{lrrrr}
\hline \multicolumn{1}{c}{ Variables } & \multicolumn{2}{c}{$\begin{array}{r}\text { CAT }+(\mathrm{n}=172) \\
\text { Mean } \pm \text { SD }\end{array}$} & $\begin{array}{c}\text { CAT }-(\mathrm{n}=112) \\
\text { Mean } \pm \text { SD }\end{array}$ & Significance \\
\hline Kcal/day & $2448.40 \pm 945.72$ & $2453.87 \pm 901.88$ & 0,96 \\
Kcal/Kg/day & $32.27 \pm$ & 11.53 & $33.30 \pm 10.35$ & 0,44 \\
Protein: $\mathrm{g} / \mathrm{Kg} /$ dia & $1.66 \pm$ & 0.70 & $1.67 \pm 0.61$ & 0,89 \\
CHO: $\mathrm{g} / \mathrm{Kg} /$ dia & $3.75 \pm$ & 1.47 & $3.94 \pm 1.43$ & 0,29 \\
Lipids: $\mathrm{g} / \mathrm{Kg} / \mathrm{dia}$ & $1.18 \pm$ & 0.59 & $1.25 \pm 0.57$ & 0,31 \\
\hline
\end{tabular}

1 - carbohydrate

t-Test 
Table 3

Prevalence of risk factors for CHD and angiographic findings:

\begin{tabular}{|c|c|c|c|c|}
\hline Variables & $\begin{array}{r}\text { CAT }+(\mathrm{r} \\
\text { Mean } \pm\end{array}$ & $\begin{array}{l}=172) \\
\text { SD }\end{array}$ & $\begin{array}{c}\text { CAT }-(n=112) \\
\text { Mean } \pm \text { SD }\end{array}$ & Significance \\
\hline Age & $60.33 \pm$ & 11.04 & $57.45 \pm 10.82$ & 0,031 \\
\hline BMI & $27.96 \pm$ & 3.97 & $28.48 \pm 5.22$ & 0,34 \\
\hline $\begin{array}{l}\text { Abdominal } \\
\text { Circumference }\end{array}$ & $100.72 \pm$ & 9.67 & $98.70 \pm 12.42$ & 0,14 \\
\hline Hip circumference & $105.03 \pm$ & 9.0 & $105.31 \pm 11.02$ & 0,81 \\
\hline WHR & $0.95 \pm$ & 0.05 & $0.93 \pm$ & 0,002 \\
\hline Fasting glucose & $101.49 \pm$ & 43.40 & $91.13 \pm 35.71$ & 0,035 \\
\hline Total cholesterol & $202.19 \pm$ & 51.41 & $201.94 \pm 46.93$ & 0,97 \\
\hline HDL cholesterol & $42.49 \pm$ & 11.18 & $46.22 \pm 12.57$ & 0,009 \\
\hline LDL cholesterol & $132.38 \pm$ & 55.22 & $130.80 \pm \quad 46.55$ & 0,98 \\
\hline Triglycerides & $159.49 \pm$ & 90.65 & $138.16 \pm 67.87$ & 0,028 \\
\hline
\end{tabular}

Table 4

Continuous variables and sex

\begin{tabular}{|c|c|c|c|c|}
\hline Variables & $\begin{array}{c}\text { Men } \\
\text { Mean } \pm \text { SD }\end{array}$ & $\begin{array}{r}\text { Wo } \\
\text { Mear }\end{array}$ & $\begin{array}{l}\text { hen } \\
\pm \mathrm{SD}\end{array}$ & Significance \\
\hline Age & $58.5 \pm 10.3$ & $60.1 \pm$ & 12.0 & 0,23 \\
\hline BMI & $27.7 \pm 4.13$ & 28.8 & 4.90 & 0,20 \\
\hline Abdominal & $101.7 \pm 10.2$ & 97.1 & 11.6 & $<0,001$ \\
\hline Circumference & & & & \\
\hline Hip circumference & $104.5 \pm 9.0$ & 105.9 & 10.9 & 0,26 \\
\hline WHR & $0.96 \pm 0.004$ & $0.91 \pm$ & 0.05 & $<0,001$ \\
\hline Glucose & $95.8 \pm 39.2$ & $99.8 \pm$ & 43.1 & 0,43 \\
\hline Total Cholesterol & $195.4 \pm 50.4$ & 212.2 & 46.6 & 0,005 \\
\hline HDL & $40.8 \pm 10.3$ & 48.7 & 12.4 & $<0,001$ \\
\hline LDL & $127.2 \pm 50.3$ & 138.0 & 53.5 & 0,07 \\
\hline Triglycerides & $152.3 \pm 84.3$ & $149.0 \pm$ & 81.1 & 0,74 \\
\hline Kcal/day & $2658.72 \pm 982.71$ & $2130.86 \pm$ & 730.24 & $<0,001$ \\
\hline $\mathrm{Kcal} / \mathrm{Kg} /$ day & $33.8 \pm 11.0$ & $30.9 \pm$ & 10.9 & 0,03 \\
\hline Protein: $\mathrm{g} / \mathrm{Kg} / \mathrm{dia}$ & $1.73 \pm 0.67$ & $1.54 \pm$ & 0.63 & 0,01 \\
\hline CHO: $\mathrm{g} / \mathrm{Kg} / \mathrm{dia}^{1}$ & $3.79 \pm 1.44$ & $3.87 \pm$ & 1.47 & 0,64 \\
\hline Lipids: $\mathrm{g} / \mathrm{Kg} / \mathrm{dia}$ & $1.28 \pm 0.61$ & $1.08 \pm$ & 0.51 & 0,004 \\
\hline
\end{tabular}

1- carbohydrate chi-square 
Table 5

Categorical variables and sex

\begin{tabular}{|c|c|c|c|}
\hline Variables & $\begin{array}{c}\text { Men } \\
\text { n (\%) }\end{array}$ & $\begin{array}{r}\text { Women } \\
\mathrm{n}(\%)\end{array}$ & $P$ \\
\hline $\begin{array}{l}\text { Abdominal } \\
\text { circumference }{ }^{1}\end{array}$ & $79(46.2)$ & $92(53.8)$ & $<0,001$ \\
\hline $\mathrm{WHR}^{2}$ & $121(52.4)$ & $110(47.6)$ & $<0,001$ \\
\hline \multicolumn{4}{|l|}{ BMI } \\
\hline$<25$ & $37(61.7)$ & $23(38.3)$ & 0,20 \\
\hline $25-29.9$ & $87(64.4)$ & $48(35.6)$ & 0,20 \\
\hline$>30$ & $44(52.4)$ & $40(47.6)$ & 0,20 \\
\hline DM & $39(22.7)$ & $23(20.5)$ & 0,77 \\
\hline $\mathrm{H}$ & $94(54.7)$ & $86(76.8)$ & 0,002 \\
\hline Family history & $103(59.9)$ & $82(73.2)$ & 0,029 \\
\hline Smoking & $125(72.7)$ & $39(34.8)$ & $<0,001$ \\
\hline Alcohol & $84(48.8)$ & $23(20.5)$ & $<0,001$ \\
\hline Physical activity & $111(64.5)$ & $81(72.3)$ & 0,48 \\
\hline \multicolumn{4}{|c|}{$\begin{array}{l}{ }^{1} \text { men } \geq 102 \mathrm{~cm}, \text { women } \geq 88 \mathrm{~cm} \\
{ }^{2} \text { men } \geq 0,95, \text { women } \geq 0,80 \\
\text { Chi-square }\end{array}$} \\
\hline \multicolumn{4}{|c|}{$\begin{array}{c}\text { Table } 6 \\
\text { Cox regression of selected variables }\end{array}$} \\
\hline Variables & significance & HR & IC95\% \\
\hline Male & 0,013 & 1.39 & $1.07-1.79$ \\
\hline Age & 0,004 & 1.01 & $1.00-1.02$ \\
\hline Smoking & 0,93 & 1.00 & $0,82-1.23$ \\
\hline Alcohol & 0,037 & 1.21 & $1.01-1.44$ \\
\hline WHR & 0,43 & 2.10 & $0,32-13.61$ \\
\hline Fasting glucose & 0,051 & 1.00 & $0,99-1.00$ \\
\hline HDL cholesterol & 0,19 & 1.00 & $0,98-1.00$ \\
\hline Triglycerides & 0,027 & 1.00 & $1.00-1.00$ \\
\hline
\end{tabular}

